\title{
A priori analysis of multilevel finite volume approximation of 1D convective Cahn-Hilliard equation
}

\author{
A. R. Appadu ${ }^{a}$, J. K. Djoko ${ }^{a, 1}$ and H. H. Gidey ${ }^{a, b}$ \\ ${ }^{a}$ Department of Mathematics and Applied Mathematics, University of Pretoria, Hatfield, Pretoria, 0002, South \\ Africa. \\ ${ }^{b}$ Department of Computational Sciences, Aksum University, Aksum, 1010, Ethiopia.
}

\begin{abstract}
In this work, we analyze four finite volume methods for the nonlinear convective Cahn-Hilliard equation with specified initial condition and periodic boundary conditions. The methods used are: implicit one-level, explicit onelevel, implicit multilevel and explicit multilevel finite volume methods. The existence and uniqueness of solution, convergence and stability of the finite volume solutions are proved. We compute $L_{2}$ - error and rate of convergence for all methods. We then compare the multilevel methods with the one-level methods by means of stability and CPU time. It is shown that the multilevel finite volume method is faster than the one-level method.
\end{abstract}

Key words. convective Cahn-Hilliard, existence, uniqueness, stability, convergence, finite volume, multilevel AMS subject classifications. 65M08, 65M12, 65M15, 65M22, 65N08, 65N12, 65N15, 65N22

\section{Contents}

1 Introduction 2

2 Some preliminaries and space discretization 4

3 One-level finite volume method 7

3.1 Implicit finite volume method . . . . . . . . . . . . . . . . . . . . . . . . 7

3.2 Explicit finite volume method . . . . . . . . . . . . . . . . . . . . . . . 17

4 Multilevel finite volume approximation 19

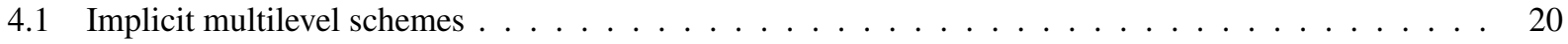

4.2 Explicit multilevel finite volume method . . . . . . . . . . . . . . . . . . . . . 24

5 Numerical results 28

5.1 Example 1 . . . . . . . . . . . . . . . . . . . . . . . . . . 28

5.2 Example $2 \ldots \ldots \ldots \ldots \ldots$

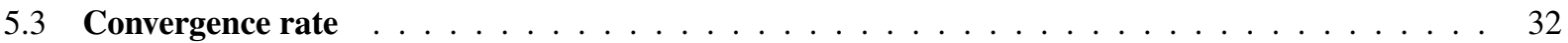

6 Conclusion 38

${ }^{1}$ Corresponding author: Jules.Djokokamdem@up.ac.za 


\section{Introduction}

In this work, we consider one-level and multilevel finite volume approximations for the one dimensional convective Cahn-Hilliard (CCH) equation:

$$
u_{t}-\gamma u u_{x}+\varepsilon^{2} u_{x x x x}=(f(u))_{x x}, x \in \mathcal{M}, t>0,
$$

with initial condition

$$
u(x, 0)=u^{0}(x), \quad x \in \overline{\mathcal{M}} .
$$

and periodic boundary conditions

$$
\frac{\partial^{j}}{\partial x^{j}} u(-L, t)=\frac{\partial^{j}}{\partial x^{j}} u(L, t), \quad j=0,1,2,3 \text { and } 0<t \leq T
$$

where

$$
f(u)=u^{3}-u,
$$

$\gamma$ is the driving force, $\mathcal{M}=(-L, L), u^{0} \in L^{2}(\mathcal{M})$ and $\varepsilon$ is a dimensionless interfacial width.

This equation is a successful model for the description of several physical phenomena: spinodal decomposition of phase separating systems in an external field, phase transition observed in alloys, glasses, polymer solution and binary liquid mixtures, faceting of growing thermodynamically unstable surfaces (see [7], [8], and the references cited therein).

The $\mathrm{CCH}$ equation is on one side related to the Cahn-Hilliard $(\mathrm{CH})$ equation and on the other side it is related to the Kuramato-Sivashinky (KS) equation, i.e., for small driving force $\gamma \rightarrow 0$, Eq. (1.1) behaves as the CH equation and for large $\gamma \rightarrow \infty$, the transformation $u \rightarrow \frac{u}{\gamma}$ reduces Eq. (1.1) to KS equation [2, 7, 5, 11]. When $\gamma \simeq 1$, the morphologies of the solutions do not coarsen as time progresses but rather display periodic patterns [11].

The CCH equation, Eq. (1.1), has been studied by several researchers, theoretically and numerically. Analytical solutions have been obtained for a single interface in the presence of the driving force, i.e $\gamma \neq 0$, in an infinite system [21]. The effect of this driving force on the coarsening dynamics of the one-dimensional Cahn-Hilliard equation at $T=0$ has been studied by Emmott and Bray [22] when $\varepsilon=1$. They observed that the driving force $\gamma$ has an asymmetric effect on the solution of a single stationary domain wall. They also noted that the behavior of the kink anti-kink pair (bubble) depends on $\gamma^{-1}$ and the separation of the interfaces. Later, Golovin et al. [7] demonstrated numerically that the one-dimensional convective Cahn-Hilliard equation exhibit a transition from coarsening to chaotic behaviour as $\gamma$ increases. The presence of the driving force elucidates a fundamental asymmetry between kinks and anti-kinks which is not present in the Cahn-Hilliard theory [5]. In Podolny et al. [8], the dynamics of domain walls (kinks) governed by the convective Cahn-Hilliard equation is studied by means of asymptotic and numerical methods. The bifurcations of stationary solutions for different values of $\gamma$ with $\varepsilon=1$ has been studied by Zaks et. al [6]. Eden and Kalantarov [2] proved the existence of compact attractor and a finite inertial manifold that contains it and X. Zhao and C. Liu [9] proved the existence of optimal solutions for the one dimensional convective Cahn-Hilliard Equations. Aderogba et. al. [1] solved the CCH equation numerically using fractional step-splitting methods for $\gamma=0.1$ and $\varepsilon=1$. They observe that the solution coarsens as $t$ progresses and they tested numerically the transition of $\mathrm{CCH}$ equation from coarsening to an order less pattern as $\gamma$ increases, which is the behavior of Kuramoto Sivashinsky equation.

Note that from (1.1)-(1.3), for $j=0$, we have

$$
\int_{-L}^{L} u(x, t) d x=\int_{-L}^{L} u^{0}(x) d x, \forall t
$$


Hence for the analysis of (1.1)-(1.3), it is important to assume that

$$
\int_{-L}^{L} u^{0}(x) d x=0
$$

In this work based on [4], we focus on the solution of the one dimensional CCH equation, with $\gamma=1$, using multilevel finite volume discretization. In many important phenomena (turbulence, excursion, etc) the solutions involves multi-scale analysis. Hence a reliable simulation requires a large number of degrees of freedom, which increases the calculation costs. Multilevel simulation in which the principle rely on the separation of scales are therefore one possibility to describe these phenomena. Early contribution include $[16,17,18,20]$, but there is now a vast literature in this research direction. It is worth mentioning that though the approach may differ from researchers to researchers, the common feature remains the same: the separation of scales.

The multilevel method we discus in this paper was formulated by Bousquet and co-workers in [4], in which a hierarchical multilevel finite volume discretization is analyzed. The contribution in [4] is a followup of ideas started in $[14,19]$. Our motivation in this work are as follows: formulate, analyze and implement the multilevel approach advocate in [4] for nonlinear partial differential equation with high order derivative. In [4], the shallow water equations in which the convective term has been linearized around a constant flow is discussed, but here we are interested in a partial differential equation containing the full convective expression $u u_{x}$ and the fact that

$$
\int_{-L}^{L} u u_{x} u d x=0
$$

plays a crucial role in designing reliable schemes. Hence when discretizing (1.1), it is desirable to approximate $u u_{x}$ in such a way that the discrete counter part of (1.4) hold. Thirdly we would like to formulate linear schemes, even though one has a nonlinear problem. Of course, one of the difficulties is that one has a nonlinear problem but one is interested in designing linear schemes. Thanks to Mickens [23], we achieve that. In particular, we approximate the nonlinear term $u u_{x}$ in such a way that the discrete analogue of property (1.4) hold.

The schemes we construct are easy to implement and are respectively called:

(a) linear implicit multilevel approximation

(b) explicit multilevel approximation.

For the sake of comparison, we also formulate two one-level methods associated to the multilevel methods. After the construction of the new schemes, we show the existence and uniqueness of the solutions. At this step, we should bear in mind that since we are dealing with linear equations in finite dimension, existence of solutions is equivalent to uniqueness. Thus we provide conditions under which there is one solution. The third contribution of this work is the stability of the new schemes. Indeed, we show that the implicit multilevel method is conditionally stable with region of stability smaller than one obtained if the one level implicit method is considered on the fine mesh. For the explicit methods we provide conditions under which the multilevel method has the same region of stability as and is less restrictive than that of the one-level method on the fine mesh. The fourth contribution of our study is the convergence analysis of the implicit methods. Indeed, we show that the implicit methods are first order accurate in time and second order accurate in space.

The last contribution of this work are numerical results. Indeed, we confirm all the theoretical findings. It is validated numerically that the implicit methods are indeed second order in space. Next we demonstrate that in all numerical test, the CPU time of the multilevel method is smaller than the one level methods on the fine mesh. Our contribution can be regarded as extension to the works $[4,19]$. Indeed, in the latter $1 \mathrm{D}$ advection equation is analyzed and 2D shallow water linearized around a constant flow is proposed and implemented. In contrast, in our work we tackle fourth order nonlinear partial differential equations. One of the challenge as mentioned earlier is to discretize the nonlinear term $u u_{x}$ in a linear way while maintaining basic properties, and as a consequence saving computational time.

The rest of this work is organized as follows: in the next section, we recall some preliminaries and introduce some standard notations. We also discuss in Section 2 some properties of difference operators and the discrete analogue 
of $L_{2}$ space. In Sections 3 and 4, one level and multilevel finite volume approximations are constructed and the stability and convergence of these methods are analyzed. Numerical simulations that confirm the theoretical work are considered in Section 5 and some conclusions are given in Section 6.

\section{Some preliminaries and space discretization}

In this section, we recall some preliminaries which are helpful to our discussion and we present the space discretization in an interval. Unlike finite difference methods in which the equation is approximated at the grid points (point wise approximation), the finite volume method is based on the integral formulation, i.e., based on subdividing the spatial domain into intervals called grid cells and approximating the integral over each of these grid cells (for more details see [26]). Finite volume is in between finite element and finite difference, hence take the good qualities of these methods, in particular less regularity is required for convergence as compared to finite difference. Our work can be regarded as an extension of the works of Bousquet et al. [4] in the sense that we incorporate higher order derivative and deal directly with a nonlinear problem.

To develop finite volume approximations we first introduce some standard notations and results.

We partition $\mathcal{M}$ into $N$ cells (intervals) $\left(k_{i}\right)_{1 \leq i \leq N}$ of uniform length $\Delta x$ with $N \Delta x=2 L$. For $0 \leq i \leq N$

$$
x_{i+1 / 2}=i \Delta x-L, \text { so that } k_{i}=\left(x_{i-1 / 2}, x_{i+1 / 2}\right) .
$$

We also consider the center of each cell:

$$
x_{i}=\frac{x_{i-1 / 2}+x_{i+1 / 2}}{2}=(i-1) \Delta x+\frac{\Delta x}{2}-L, 1 \leq i \leq N .
$$

We denote $u_{i}^{n}$ as the approximate solution to the cell average of the true solution at $t_{n}=n \Delta t$ with $M \Delta t=T$, $0 \leq n \leq M$, i.e.,

$$
u_{i}^{n} \approx \frac{1}{\Delta x} \int_{k_{i}} u\left(x, t_{n}\right) d x, 1 \leq i \leq N,
$$

which is obtained recursively by starting with the sequence $u_{i}^{0}$ given by

$$
u_{i}^{0}=\frac{1}{\Delta x} \int_{k_{i}} u^{0}(x) d x, 1 \leq i \leq N
$$

Define the space $\mathcal{H}_{h}$ as

$$
\mathcal{H}_{h}=\left\{\mathbf{u}=\left(u_{i}\right), u_{i} \in \mathbb{R} \mid u_{i+N}=u_{i}, i \in \mathbb{Z} \text { and } \sum_{i=1}^{N} u_{i}=0\right\} .
$$

We equip $\mathcal{H}_{h}$ with the inner product

$$
(\mathbf{u}, \mathbf{v})_{h}=\Delta x \sum_{i=1}^{N} u_{i} v_{i}
$$

and discrete $L^{2}$ norm

$$
\|\mathbf{u}\|_{h}=\left(\Delta x \sum_{i=1}^{N} u_{i}^{2}\right)^{1 / 2}
$$


For $\mathbf{u}, \mathbf{v} \in \mathcal{H}_{h}$, we introduce the following difference operators:

$$
\begin{aligned}
& \nabla_{h}^{-} u_{i}=\frac{1}{\Delta x}\left(u_{i}-u_{i-1}\right), \nabla_{h}^{+} u_{i}=\frac{1}{\Delta x}\left(u_{i+1}-u_{i}\right), \\
& \Delta_{h} u_{i}=\nabla_{h}^{+}\left(\nabla_{h}^{-} u_{i}\right)=\frac{1}{\Delta x^{2}}\left(u_{i+1}-2 u_{i}+u_{i-1}\right), \\
& \Delta_{h}^{2} u_{i}=\Delta_{h}\left(\Delta_{h} u_{i}\right)=\frac{1}{\Delta x^{2}}\left(\Delta_{h} u_{i+1}-2 \Delta_{h} u_{i}+\Delta_{h} u_{i-1}\right), \\
& \nabla_{h}^{+}\left(u_{i} v_{i}\right)=\left(\nabla_{h}^{+} u_{i}\right) v_{i+1}+u_{i}\left(\nabla_{h}^{+} v_{i}\right), \\
& \nabla_{h}^{-}\left(u_{i} v_{i}\right)=\left(\nabla_{h}^{-} u_{i}\right) v_{i-1}+u_{i}\left(\nabla_{h}^{-} v_{i}\right) .
\end{aligned}
$$

From the definitions of $\mathcal{H}_{h}$ and the discrete operators, one obtains:

Lemma 2.1. Let $\boldsymbol{u}, \boldsymbol{w} \in \mathcal{H}_{h}$ then

$$
\sum_{i=1}^{N} w_{i} \nabla_{h}^{+} u_{i}=-\sum_{i=1}^{N} u_{i} \nabla_{h}^{-} w_{i} .
$$

We define the following norms and semi-norms on $\mathcal{H}_{h}$

$$
\begin{aligned}
|\mathbf{u}|_{1, h} & =\left(\Delta x \sum_{i=1}^{N}\left(\nabla_{h}^{-} u_{i}\right)^{2}\right)^{\frac{1}{2}}, \\
|\mathbf{u}|_{2, h} & =\left(\Delta x \sum_{i=1}^{N}\left(\Delta_{h} u_{i}\right)^{2}\right)^{\frac{1}{2}}, \\
\|\mathbf{u}\|_{\infty, h} & =\max _{1 \leq i \leq N}\left|u_{i}\right| .
\end{aligned}
$$

In Eq. (2.1), $\nabla_{h}^{-}$can be replaced by $\nabla_{h}^{+}$. Using Eqs. (2.1)-(2.2), we obtain

$$
|\mathbf{u}|_{1, h} \leq \frac{2}{\Delta x}\|\mathbf{u}\|_{h}, \quad|\mathbf{u}|_{2, h} \leq \frac{4}{\Delta x^{2}}\|\mathbf{u}\|_{h}
$$

and the following are obtained by direct computations

$$
\|\mathbf{u}\|_{h}^{2} \leq 2 L\|\mathbf{u}\|_{\infty, h}^{2}, \quad\|\mathbf{u}\|_{\infty, h}^{2} \leq \frac{1}{\Delta x}\|\mathbf{u}\|_{h}^{2} .
$$

We recall following identities and inequalities which are helpful for the later discussion.

- For any $\mathbf{u}, \mathbf{v} \in \mathcal{H}_{h}$

$$
\begin{aligned}
& 2(\mathbf{u}-\mathbf{v}, \mathbf{u})_{h}=\|\mathbf{u}\|_{h}^{2}-\|\mathbf{v}\|_{h}^{2}+\|\mathbf{u}-\mathbf{v}\|_{h}^{2}, \\
& 2(\mathbf{u}-\mathbf{v}, \mathbf{v})_{h}=\|\mathbf{u}\|_{h}^{2}-\|\mathbf{v}\|_{h}^{2}-\|\mathbf{u}-\mathbf{v}\|_{h}^{2} .
\end{aligned}
$$

- For $x \in\left[0, \frac{1}{2}\right]$,

$$
\left(\frac{1}{2}\right)^{2 x} \leq 1-x
$$

- Young's inequality: For any $a, b \in \mathbb{R}$ and any $\delta>0$, we have

$$
a b \leq \frac{\delta}{2} a^{2}+\frac{1}{2 \delta} b^{2} .
$$


- Cauchy-Schwarz's inequality: For $N \in \mathbb{N}$

$$
\sum_{i=1}^{N} a_{i} b_{i} \leq\left(\sum_{i=1}^{N} a_{i}^{2}\right)^{1 / 2}\left(\sum_{i=1}^{N} b_{i}^{2}\right)^{1 / 2} .
$$

We also note that for $\mathbf{u} \in \mathcal{H}_{h}$, then the discrete Poincaré's inequality holds: that is, there exist $\eta>0$ independent of the spatial step size such that

$$
\eta\|\mathbf{u}\|_{h} \leq|\mathbf{u}|_{1, h} .
$$

Before the formulation of the finite volume discretization of (1.1)-(1.3), one observes that

$$
\int_{-L}^{L} u^{2} u_{x} d x=0
$$

Hence, when discretizing (1.1), it is desirable to approximate $u u_{x}$ in such a way that the discrete counter part of (2.11) hold (see $[15,24,25])$. In this regard, in [3], $u u_{x}$ was approximated using finite difference scheme by a linear term which moreover replicate (2.11) in the discrete setting. In the same spirit, one introduces a bilinear map $C_{h}$ given as follows

$$
\begin{aligned}
& C_{h}: \mathcal{H}_{h} \times \mathcal{H}_{h} \rightarrow \mathbb{R}^{N}, \\
& \left(C_{h}(\mathbf{u}, \mathbf{v})\right)_{i}=\alpha_{1}\left[u_{i} \nabla_{h}^{+} v_{i}+v_{i} \nabla_{h}^{-} u_{i}+v_{i+1} \nabla_{h}^{+} u_{i}\right]+\alpha_{2}\left[u_{i} \nabla_{h}^{-} v_{i}+v_{i} \nabla_{h}^{+} u_{i}+v_{i-1} \nabla_{h}^{-} u_{i}\right],
\end{aligned}
$$

where $\alpha_{1}, \alpha_{2} \in \mathbb{R}$ as we will see play a crucial role in obtaining consistency of the approximation. One readily check that the property

$$
\left(C_{h}(\mathbf{u}, \mathbf{v}), \mathbf{u}\right)_{h}=0,
$$

holds for any $\mathbf{u}, \mathbf{v} \in \mathcal{H}_{h}$.

Lemma 2.2. For $\boldsymbol{u} \in \mathcal{H}_{h}$, we have

$$
|\boldsymbol{u}|_{1, h}^{2} \leq|\boldsymbol{u}|_{2, h}\|\boldsymbol{u}\|_{h} .
$$

Proof. Applying Lemma 2.1 for all $\mathbf{u} \in \mathcal{H}_{h}$, we have

$$
|\mathbf{u}|_{1, h}^{2}=\sum_{i=1}^{N}\left(\nabla_{h}^{-} u_{i}\right)\left(\nabla_{h}^{-} u_{i}\right)=-\sum_{i=1}^{N} u_{i}\left(\Delta_{h} u_{i}\right),
$$

which by Cauchy-Schwarz's inequality gives

$$
|\mathbf{u}|_{1, h}^{2} \leq\left\|\Delta_{h} \mathbf{u}\right\|_{h}\|\mathbf{u}\|_{h}=|\mathbf{u}|_{2, h}\|\mathbf{u}\|_{h} .
$$

For $\mathbf{u} \in \in \mathcal{H}_{h}$, Lemma 2.2 and Young's inequality implies the existence of $\eta$, positive constant independent of $\Delta x$ such that

$$
\eta|\mathbf{u}|_{1, h} \leq|\mathbf{u}|_{2, h}
$$


Lemma 2.3. For $\boldsymbol{u}, \boldsymbol{v} \in \mathcal{H}_{h}$, we have

$$
\sum_{i=1}^{N} \Delta x \nabla_{h}^{+}\left[\varphi_{i-\frac{1}{2}} \nabla_{h}^{-} u_{i}\right] u_{i} \leq\|\boldsymbol{u}\|_{1, h}^{2}
$$

where

$$
\varphi_{i-\frac{1}{2}}=\frac{f^{\prime}\left(v_{i}\right)+f^{\prime}\left(v_{i-1}\right)}{2} .
$$

Proof. For $\mathbf{u}, \mathbf{v} \in \mathcal{H}_{h}$ from lemma 2.1, we have

$$
\sum_{i=1}^{N} \Delta x \nabla_{h}^{+}\left[\varphi_{i-\frac{1}{2}} \nabla_{h}^{-} u_{i}\right] u_{i}=-\Delta x \sum_{i=1}^{N} \varphi_{i-\frac{1}{2}}\left(\nabla_{h}^{-} u_{i}\right)^{2}
$$

From the definition of $\varphi_{i-1 / 2}$, we get

$$
\Delta x \sum_{i=1}^{N} \nabla_{h}^{+}\left[\varphi_{i-\frac{1}{2}} \nabla_{h}^{-} u_{i}\right] u_{i}=-\frac{3 \Delta x}{2} \sum_{i=1}^{N}\left(\left(v_{i}\right)^{2}+\left(v_{i-1}\right)^{2}\right)\left(\nabla_{h}^{-} u_{i}\right)^{2}+\Delta x \sum_{i=1}^{N}\left(\nabla_{h}^{-} u_{i}\right)^{2} .
$$

Since the first expression of the right hand side of Eq. (2.16) is negative, we obtain

$$
\Delta x \sum_{i=1}^{N} \nabla_{h}^{+}\left[\varphi_{i-\frac{1}{2}} \nabla_{h}^{-} u_{i}\right] u_{i} \leq \Delta x \sum_{i=1}^{N}\left(\nabla_{h}^{-} u_{i}\right)^{2}=|\mathbf{u}|_{1, h}^{2} .
$$

\section{One-level finite volume method}

In this section, we present two traditional one level finite volume methods: namely implicit finite volume method and explicit finite volume method. For the implicit method the existence of solution has been proved and convergence analysis has been studied. The stability analysis has been examined for both schemes. For both methods thirteen point stencils are used to approximate (1.1)-(1.3). The introduction of these classical schemes are important at least for two reasons:

(a) comparison with multilevel methods.

(b) these schemes that are categorized as classical present significant challenges for their analysis as we will see.

\subsection{Implicit finite volume method}

This method approximates the nonlinear term $u u_{x}$ at time $t=(n+1) \Delta t$ by the relation:

$$
\begin{aligned}
\left.u u_{x}\right|_{i} ^{n+1} \simeq\left(C_{h}\left(\mathbf{u}^{n+1}, \tilde{\mathbf{u}}^{n}\right)\right)_{i}= & \alpha_{1}\left[u_{i}^{n+1} \nabla_{h}^{+} \tilde{u}_{i}+\tilde{u}_{i} \nabla_{h}^{-} u_{i}^{n+1}+\tilde{u}_{i+1} \nabla_{h}^{+} u_{i}^{n+1}\right] \\
& +\alpha_{2}\left[u_{i}^{n+1} \nabla_{h}^{-} \tilde{u}_{i}+\tilde{u}_{i} \nabla_{h}^{+} u_{i}^{n+1}+\tilde{u}_{i-1} \nabla_{h}^{-} u_{i}^{n+1}\right],
\end{aligned}
$$

$\tilde{\mathbf{u}}^{n}$ is an approximation of $\mathbf{u}^{n+1}$ given by

$$
\tilde{\mathbf{u}}^{n}=a_{1} \mathbf{u}^{n}+a_{2} \mathbf{u}^{n-1}+a_{3} \mathbf{u}^{n-2}+\cdots+a_{m_{0}} \mathbf{u}^{n-m_{0}+1},
$$

where $a_{1}, a_{2}, \ldots$, and $a_{m_{0}}$ are constant coefficients that determine the approximation with

$$
3\left(\alpha_{1}+\alpha_{2}\right)\left(a_{1}+a_{2}+\cdots+a_{m_{0}}\right)=1 .
$$


For $m<m_{0}-1$, the term $\tilde{\mathbf{u}}^{n}$ is given by the relation

$$
\tilde{\mathbf{u}}^{m}=\mathbf{u}^{m}
$$

The nonlinear term on the right hand side of (1.1) is also approximated by a linear term given by the relation

$$
\Delta_{h} f\left(u_{i}^{n+1}\right) \simeq \nabla_{h}^{+}\left[\varphi_{i-\frac{1}{2}}^{n} \nabla_{h}^{-} u_{i}^{n+1}\right]
$$

where

$$
\varphi_{i-\frac{1}{2}}^{n}=\frac{f^{\prime}\left(u_{i}^{n}\right)+f^{\prime}\left(u_{i-1}^{n}\right)}{2} .
$$

The fourth order derivative is discretized using the central space difference method and together with (3.1) and (3.5), the full discretization of Eqs. (1.1)-(1.3) is given as follows:

$$
\begin{aligned}
& \frac{u_{i}^{n+1}-u_{i}^{n}}{\Delta t}-C_{h}\left(\mathbf{u}^{n+1}, \tilde{\mathbf{u}}^{n}\right)_{i}+\varepsilon^{2} \Delta_{h}^{2} u_{i}^{n+1}=\nabla_{h}^{+}\left[\varphi_{i-\frac{1}{2}}^{n} \nabla_{h}^{-} u_{i}^{n+1}\right], \\
& u_{i}^{n}=u_{i+N}^{n} \\
& u_{i}^{0}=\frac{1}{\Delta x} \int_{k_{i}} u^{0}(x) d x .
\end{aligned}
$$

Remark 3.1. Using Taylor's expansion, we have

$$
\begin{aligned}
\nabla^{+}\left[u_{i-\frac{1}{2}}^{n} \nabla^{-} u_{i}^{n+1}\right] & =\left.3 u^{2} u_{x x}\right|_{i} ^{n}+\left.6 u u_{x}^{2}\right|_{i} ^{n}+\left.3 \Delta t u^{2} u_{x x t}\right|_{i} ^{n}+\left.6 \Delta t u u_{x} u_{x t}\right|_{i} ^{n}+\mathcal{O}\left(\Delta t^{2}+\Delta x^{2}\right) . \\
\left(C_{h}\left(\boldsymbol{u}^{n+1}, \tilde{\boldsymbol{u}}^{n}\right)\right)_{i} & =\left.3\left(\alpha_{1}+\alpha_{2}\right)\left(a_{1}+a_{2}+\cdots+a_{m_{0}}\right) u u_{x}\right|_{i} ^{n}+\mathcal{O}\left(\Delta t+\Delta x^{2}\right) .
\end{aligned}
$$

From (3.7) and (3.8), we can see that the approximations (3.5) and (3.1) are consistent provided (3.3) holds for the latter. One can easily show that the other terms in Eq. (3.6a) are consistent with their corresponding terms.

Hence the finite volume method (3.6) is consistent.

The problem (3.6) is a linear problem in finite dimensional space, hence existence of solution is equivalent to uniqueness of solution. Thus, we only show that the approximations $\mathbf{u}^{1}, \mathbf{u}^{2}, \ldots, \mathbf{u}^{M}$ satisfying (3.6a)-(3.6c), are unique.

Theorem 3.1. If $\Delta t \leq 4 \varepsilon^{2}$, then the approximate solution $\boldsymbol{u}^{n}$ of (3.6) is unique.

Proof. For $n=0,1, \ldots, M$, let $\mathbf{v}^{n}$ and let $\mathbf{v}^{n}$ are two solutions of (3.6) such that $\mathbf{u}^{0}=\mathbf{v}^{0}$. Let $\mathbf{z}^{n}=\mathbf{u}^{n}-\mathbf{v}^{n}$. Clearly $\mathbf{z}^{0}=0$. We would prove by induction on $n$ for $n=1, \ldots, M$ that $\mathbf{z}^{n}=0$. One obtains

$$
\begin{aligned}
\frac{1}{\Delta t}\left(z_{i}^{n+1}-z_{i}^{n}\right)-\left(C_{h}\left(\mathbf{u}^{n+1}, \tilde{\mathbf{u}}^{n}\right)\right)_{i}+\left(C_{h}\left(\mathbf{v}^{n+1}, \tilde{\mathbf{v}}^{n}\right)\right)_{i}+\varepsilon^{2} \Delta_{h}^{2} z_{i}^{n+1}= & \nabla_{h}^{+}\left[\varphi_{i-\frac{1}{2}}^{n} \nabla_{h}^{-} u_{i}^{n+1}\right] \\
& -\nabla_{h}^{+}\left[\psi_{i-\frac{1}{2}}^{n} \nabla_{h}^{-} v_{i}^{n+1}\right]
\end{aligned}
$$

for $i=1, \ldots N$, where

$$
\psi_{i-\frac{1}{2}}^{n}=\frac{f^{\prime}\left(v_{i}^{n}\right)+f^{\prime}\left(v_{i-1}^{n}\right)}{2} .
$$

We assume that $\mathbf{z}^{n}=0$ and using (2.13), (3.9) becomes

$$
\frac{1}{\Delta t}\left(z_{i}^{n+1}\right)-\left(C_{h}\left(\mathbf{z}^{n+1}, \tilde{\mathbf{u}}^{n}\right)\right)_{i}+\varepsilon^{2} \Delta_{h}^{2} z_{i}^{n+1}=\nabla_{h}^{+}\left(\varphi_{i-\frac{1}{2}}^{n} \nabla_{h}^{-} z_{i}^{n+1}\right) .
$$


Multiplying Eq. (3.10) by $\Delta t \Delta x z_{i}^{n+1}$ and adding the resulting equalities for $i=1, \ldots, N$, we obtain

$$
\left\|\mathbf{z}^{n+1}\right\|_{h}^{2}+\Delta t \varepsilon^{2}\left|\mathbf{z}^{n+1}\right|_{2, h}^{2}=\Delta t \Delta x \sum_{i=1}^{N} \nabla_{h}^{+}\left(\varphi_{i-\frac{1}{2}}^{n} \nabla_{h}^{-} z_{i}^{n+1}\right),
$$

which after application of Lemma 2.3 gives

$$
\left\|\mathbf{z}^{n+1}\right\|_{h}^{2}+\Delta t \varepsilon^{2}\left|\mathbf{z}^{n+1}\right|_{2, h}^{2} \leq \Delta t\left|\mathbf{z}^{n+1}\right|_{1, h}^{2} .
$$

Using Lemma 2.2 and Young's inequality, (3.11) implies that

$$
\left(1-\frac{\Delta t}{4 \varepsilon^{2}}\right)\left\|\mathbf{z}^{n+1}\right\|_{h}^{2} \leq\left\|\mathbf{z}^{n}\right\|_{h}^{2}
$$

which for $\frac{\Delta t}{4 \varepsilon^{2}}<1$ gives

$$
\left\|\mathbf{z}^{n+1}\right\|_{h}^{2}=0 .
$$

Therefore, $\mathbf{z}^{n+1}=0$. This completes the proof of uniqueness and hence existence of the solution.

Theorem 3.2. The finite volume method defined by (3.6) is conditionally stable in $L^{\infty}\left(0, T ; \mathcal{H}_{h}\right)$, that is, for $\Delta t \leq \varepsilon^{2}$ and $1 \leq n \leq M$

$$
\left\|\boldsymbol{u}^{n}\right\|_{h}^{2} \leq 2^{\frac{2 T}{\varepsilon^{2}}}\left\|\boldsymbol{u}^{0}\right\|_{h}^{2} .
$$

Proof. By multiplying Eq. (3.6a) with $2 \Delta x \Delta t u_{i}^{n+1}$ and summing from $i=1$ to $N$, we obtain

$$
2 \Delta x \sum_{i=1}^{N}\left(u_{i}^{n+1}-u_{i}^{n}\right) u_{i}^{n+1}+2 \Delta t \Delta x \varepsilon^{2} \sum_{i=1}^{N} \Delta_{h}^{2} u_{i}^{n+1} u_{i}^{n+1}=2 \Delta t \Delta x \sum_{i=1}^{N} \nabla_{h}^{+}\left[\varphi_{i-\frac{1}{2}}^{n} \nabla_{h}^{-} u_{i}^{n+1}\right] u_{i}^{n+1} \text {. }
$$

Using Eqs. (2.5) and (2.1), we get

$$
\left\|\mathbf{u}^{n+1}\right\|_{h}^{2}-\left\|\mathbf{u}^{n}\right\|_{h}^{2}+\left\|\mathbf{u}^{n+1}-\mathbf{u}^{n}\right\|_{h}^{2}+2 \Delta t \varepsilon^{2}\left|\mathbf{u}^{n+1}\right|_{2, h}^{2}=2 \Delta t \Delta x \sum_{i=1}^{N} \nabla_{h}^{+}\left(\varphi_{i-\frac{1}{2}}^{n} \nabla_{h}^{-} u_{i}^{n+1}\right) u_{i}^{n+1} .
$$

Using Lemma 2.3 together with (3.12), we have

$$
\left\|\mathbf{u}^{n+1}\right\|_{h}^{2}-\left\|\mathbf{u}^{n}\right\|_{h}^{2}+\left\|\mathbf{u}^{n+1}-\mathbf{u}^{n}\right\|_{h}^{2}+2 \Delta t \varepsilon^{2}\left|\mathbf{u}^{n+1}\right|_{2, h}^{2} \leq 2 \Delta t\left|\mathbf{u}^{n+1}\right|_{1, h}^{2} .
$$

Using Lemma (2.2) and Young's inequality, (3.13) becomes

$$
\left\|\mathbf{u}^{n+1}\right\|_{h}^{2}-\left\|\mathbf{u}^{n}\right\|_{h}^{2}+\left\|\mathbf{u}^{n+1}-\mathbf{u}^{n}\right\|_{h}^{2} \leq \frac{\Delta t}{2 \varepsilon^{2}}\left\|\mathbf{u}^{n+1}\right\|_{h}^{2} .
$$

By dropping the term $\left\|\mathbf{u}^{n+1}-\mathbf{u}^{n}\right\|_{h}^{2}$, one obtains

$$
\left[1-\frac{\Delta t}{2 \varepsilon^{2}}\right]\left\|\mathbf{u}^{n+1}\right\|_{h}^{2} \leq\left\|\mathbf{u}^{n}\right\|_{h}^{2} .
$$

Based on (2.7), for $\frac{\Delta t}{2 \varepsilon^{2}} \leq \frac{1}{2}$, (3.14) gives

$$
\left\|\mathbf{u}^{n+1}\right\|_{h}^{2} \leq 2 \frac{2 \Delta t}{\varepsilon^{2}}\left\|\mathbf{u}^{n}\right\|_{h}^{2} .
$$

By induction over $n$, we obtain

$$
\left\|\mathbf{u}^{n}\right\|_{h}^{2} \leq 2 \frac{2 n \Delta t}{\varepsilon^{2}}\left\|\mathbf{u}^{0}\right\|_{h}^{2} \leq 2 \frac{2 T}{\varepsilon^{2}}\left\|\mathbf{u}^{0}\right\|_{h}^{2} .
$$

Therefore, the proof is complete. 
Theorem 3.3. Suppose that the solution $u(x, t)$ of Eqs. (1.1)-(1.3) is sufficiently smooth. Assume that $\Delta t$ and $\Delta x$ satisfy the relation (3.38) and $\Delta t<\min \left(4 \varepsilon^{2}, c\right)$, with $c$ independent of $\Delta t$ and $\Delta x$ given by (3.36). Then, the solution of the finite volume discretization (3.6) converges to the solution of Eq. (1.1) in the discrete $L^{2}$-norm with rate of convergence $\mathcal{O}\left(\Delta t+\Delta x^{2}\right)$.

Proof. Let

$$
v_{i}^{n}=\int_{x_{i-1 / 2}}^{x_{i+1 / 2}} u\left(x, t_{n}\right) d x, 1 \leq i \leq N, 0 \leq n \leq M
$$

be the cell average of the exact solution $u$ of Eqs. (1.1)-(1.3). From the smoothness of $u$

$$
v_{i}^{n}=u\left(x_{i}, t_{n}\right)+\mathcal{O}\left(\Delta x^{2}\right), 1 \leq i \leq N, 0 \leq n \leq M
$$

and denote

$$
s=\max _{-L \leq x \leq L, 0 \leq t \leq T}|u(x, t)|
$$

Making use of Taylor expansion, we obtain

$$
\begin{aligned}
& \frac{v_{i}^{n+1}-v_{i}^{n}}{\Delta t}=\left.u_{t}\right|_{i} ^{n}+\left.\frac{\Delta t}{2} v_{t t}\right|_{i} ^{n}+\mathcal{O}\left(\Delta t^{2}\right) \\
& \Delta_{h}^{2} v_{i}^{n+1}=\left.u_{x x x x}\right|_{i} ^{n}+\left.\Delta t u_{x x x x t}\right|_{i} ^{n}+\mathcal{O}\left(\Delta t^{2}+\Delta x^{2}\right) \\
& \Delta_{h} v_{i}^{n+1}=\left.u_{x x}\right|_{i} ^{n}+\left.\Delta t u_{x x t}\right|_{i} ^{n}+\mathcal{O}\left(\Delta t^{2}+\Delta x^{2}\right) \\
& \nabla^{+}\left[\psi_{i-\frac{1}{2}}^{n} \nabla^{-} v_{i}^{n+1}\right]=\left.3 u^{2} u_{x x}\right|_{i} ^{n}+\left.6 u u_{x}^{2}\right|_{i} ^{n}+\left.3 \Delta t u^{2} u_{x x t}\right|_{i} ^{n}+\left.6 \Delta t u u_{x} u_{x t}\right|_{i} ^{n}+\mathcal{O}\left(\Delta t^{2}+\Delta x^{2}\right) . \\
&\left(C_{h}\left(\boldsymbol{v}^{n+1}, \tilde{\boldsymbol{v}}^{n}\right)\right)_{i}=3\left(\alpha_{1}+\alpha_{2}\right)\left.\left(a_{1}+a_{2}+\cdots+a_{m_{0}}\right) u u_{x}\right|_{i} ^{n}+\left.\frac{\Delta x}{2}\left(\alpha_{1}-\alpha_{2}\right)\left(a_{1}+a_{2}+\cdots+a_{m_{0}}\right) u u_{x x}\right|_{i} ^{n} \\
&+\left.\Delta x\left(\alpha_{1}-\alpha_{2}\right)\left(a_{1}+a_{2}+\cdots+a_{m_{0}}\right) u_{x}^{2}\right|_{i} ^{n}+\mathcal{O}\left(\Delta t+\Delta x^{2}\right)
\end{aligned}
$$

For $\alpha_{1}=\alpha_{2}$, we get

$$
C_{h}\left(\boldsymbol{v}^{n+1}, \tilde{\boldsymbol{v}}^{n}\right)=\left.u u_{x}\right|_{i} ^{n}+\mathcal{O}\left(\Delta t+\Delta x^{2}\right)
$$

all the terms are second order accurate in space.

Thus, in this study, we consider $\alpha_{1}=\alpha_{2}$ and combining all the terms, we obtain

$$
\left\{\begin{array}{l}
\frac{v_{i}^{n+1}-v_{i}^{n}}{\Delta t}-\left(C_{h}\left(\boldsymbol{v}^{n+1}, \tilde{\boldsymbol{v}}^{n}\right)\right)_{i}+\varepsilon^{2} \Delta_{h}^{2} v_{i}^{n+1}=\nabla_{h}^{+}\left[\psi_{i-\frac{1}{2}}^{n} \nabla_{h}^{-} v_{i}^{n+1}\right]+r_{i}^{n}, \\
v_{i}^{0}=u^{0}(x),
\end{array}\right.
$$

where $\psi_{i-\frac{1}{2}}^{n}=\frac{f^{\prime}\left(v_{i}^{n}\right)+f^{\prime}\left(v_{i-1}^{n}\right)}{2}$ and $r_{i}^{n}$ is the truncation error of the finite volume discretization (3.6) for $0 \leq n \leq$ $M-1$ and $1 \leq i \leq N$. There exists a positive constant $c_{1}$ such that

$$
\max _{i, n}\left|r_{i}^{n}\right| \leq c_{1}\left(\Delta t+\Delta x^{2}\right), 0 \leq n \leq M-1,1 \leq i \leq N .
$$

Let $\mathbf{e}^{n}=\boldsymbol{v}^{n}-\mathbf{u}^{n}, 0 \leq n \leq M$, where $u_{i}^{n}$ is the solution of Eqs. (3.6a)-(3.6c). It is clear that $\mathbf{e}^{n} \in \mathcal{H}_{h}$ and $\mathbf{e}^{0}=0$. Substituting $\mathbf{u}^{n}=\boldsymbol{v}^{n}-\mathbf{e}^{n}$ into Eq. (3.6a), we obtain

$$
\begin{aligned}
\frac{v_{i}^{n+1}-v_{i}^{n}}{\Delta t}-\left(C_{h}\left(\boldsymbol{v}^{n+1}, \tilde{\boldsymbol{v}}^{n}\right)\right)_{i}+\varepsilon^{2} \Delta_{h}^{2} v_{i}^{n+1}-\nabla_{h}^{+}\left[\psi_{i-\frac{1}{2}}^{n} \nabla_{h}^{-} v_{i}^{n+1}\right] & \begin{aligned}
= & \frac{e_{i}^{n+1}-e_{i}^{n}}{\Delta t}-\left(C_{h}\left(\mathbf{e}^{n+1}, \tilde{\boldsymbol{v}}^{n}-\tilde{\mathbf{e}}^{n}\right)\right)_{i}+\varepsilon^{2} \Delta_{h}^{2} e_{i}^{n+1}-\nabla_{h}^{+}\left[\varphi_{i-\frac{1}{2}}^{n} \nabla_{h}^{-} e_{i}^{n+1}\right] \\
& \quad-\nabla_{h}^{+}\left[\left(\psi_{i-\frac{1}{2}}^{n}-\varphi_{i-\frac{1}{2}}^{n}\right) \nabla_{h}^{-} v_{i}^{n+1}\right]-\left(C_{h}\left(\boldsymbol{v}^{n+1}, \tilde{\mathbf{e}}^{n}\right)\right)_{i} .
\end{aligned}
\end{aligned}
$$


Using (3.15), Eq. (3.17) becomes

$$
\begin{aligned}
\frac{e_{i}^{n+1}-e_{i}^{n}}{\Delta t}-\left(C_{h}\left(\mathbf{e}^{n+1}, \tilde{\boldsymbol{v}}^{n}-\tilde{\mathbf{e}}^{n}\right)\right)_{i}+ & \varepsilon^{2} \Delta_{h}^{2} e_{i}^{n+1}=\nabla_{h}^{+}\left[\varphi_{i-\frac{1}{2}}^{n} \nabla_{h}^{-} e_{i}^{n+1}\right] \\
& +\nabla_{h}^{+}\left[\left(\psi_{i-\frac{1}{2}}^{n}-\varphi_{i-\frac{1}{2}}^{n}\right) \nabla_{h}^{-} v_{i}^{n+1}\right]+\left(C_{h}\left(\boldsymbol{v}^{n+1}, \tilde{\mathbf{e}}^{n}\right)\right)_{i}+r_{i}^{n}
\end{aligned}
$$

Multiplying Eq. (3.18) by $2 \Delta t \Delta x e_{i}^{n+1}$ and summing the corresponding equalities for $i=1, \ldots N$, we obtain

$$
\begin{aligned}
2\left(\mathbf{e}^{n+1}-\mathbf{e}^{n}, \mathbf{e}^{n+1}\right)_{h}+2 \Delta t \varepsilon^{2}\left\|\mathbf{e}^{n+1}\right\|_{2, h}^{2}= & 2 \Delta t \Delta x \sum_{i=1}^{N} \nabla_{h}^{+}\left[\varphi_{i-\frac{1}{2}}^{n} \nabla_{h}^{-} e_{i}^{n+1}\right] e_{i}^{n+1} \\
& +2 \Delta t \Delta x \sum_{i=1}^{N} \nabla_{h}^{+}\left[\left(\psi_{i-\frac{1}{2}}^{n}-\varphi_{i-\frac{1}{2}}^{n}\right) \nabla_{h}^{-} v_{i}^{n+1}\right] e_{i}^{n+1} \\
& +2 \Delta t\left(C_{h}\left(\boldsymbol{v}^{n+1}, \tilde{\mathbf{e}}^{n}\right), \mathbf{e}^{n+1}\right)_{h}+2 k\left(\mathbf{r}^{n}, \mathbf{e}^{n+1}\right)_{h} .
\end{aligned}
$$

Using (2.5) and Lemmas 2.1, Eq. (3.19) yields

$$
\begin{aligned}
\left\|\mathbf{e}^{n+1}\right\|_{h}^{2}-\left\|\mathbf{e}^{n}\right\|_{h}^{2}+\left\|\mathbf{e}^{n+1}-\mathbf{e}^{n}\right\|_{h}^{2}+2 \Delta t \varepsilon^{2}\left|\mathbf{e}^{n+1}\right|_{2, h}^{2} \leq & 2 \Delta t\left|\mathbf{e}^{n+1}\right|_{1, h}^{2} \\
& -6 \Delta t \Delta x \sum_{i=1}^{N}\left[\left(v_{i}^{n} e_{i}^{n}+v_{i-1}^{n} e_{i-1}^{n}\right) \nabla_{h}^{-} v_{i}^{n+1} \nabla_{h}^{-} e_{i}^{n+1}\right] \\
& +3 \Delta t \Delta x \sum_{i=1}^{N}\left[\left[\left(e_{i}^{n}\right)^{2}+\left(e_{i-1}^{n}\right)^{2}\right] \nabla_{h}^{-} v_{i}^{n+1} \nabla_{h}^{-} e_{i}^{n+1}\right] \\
& +2 \Delta t\left(C_{h}\left(\boldsymbol{v}^{n+1}, \tilde{\mathbf{e}}^{n}\right), \mathbf{e}^{n+1}\right)_{h}+2 \Delta t\left(\mathbf{r}^{n}, \mathbf{e}^{n+1}\right)_{h} . \quad(3.20)
\end{aligned}
$$

We estimate each of the terms on the right hand side of (3.20) as follows:

$$
\begin{aligned}
-2 \Delta t & \Delta x \sum_{i=1}^{N}\left(3\left(v_{i}^{n} e_{i}^{n}+v_{i-1}^{n} e_{i-1}^{n}\right)\right) \nabla_{h}^{-} v_{i}^{n+1} \nabla_{h}^{-} e_{i}^{n+1} \\
\leq & 6 \Delta t\left[\left(\max _{1 \leq i \leq N}\left|v_{i}^{n}\right|\right)\left(\max _{1 \leq i \leq N}\left|\nabla_{h}^{-} v_{i}^{n}\right|\right)\right]\left(\Delta x \sum_{i=1}^{N}\left|e_{i}^{n}\right|\left|\nabla_{h}^{-} e_{i}^{n+1}\right|+\left|e_{i-1}^{n}\right|\left|\nabla_{h}^{-} e_{i}^{n+1}\right|\right)
\end{aligned}
$$

Since $u$ is smooth then there exists constant $c_{2}$ such that

$$
\max _{1 \leq i \leq N}\left|\nabla_{h}^{-} v_{i}^{n+1}\right| \leq c_{2} \quad \forall n=0,1, \cdots, M-1 .
$$

Thus,

$$
\begin{aligned}
-2 \Delta t \Delta x \sum_{i=1}^{N} 3\left(v_{i}^{n} e_{i}^{n}+v_{i-1}^{n} e_{i-1}^{n}\right) \nabla_{h}^{-} v_{i}^{n+1} \nabla_{h}^{-} e_{i}^{n+1} & \leq 6 s c_{2} \Delta t\left(\Delta x \sum_{i=1}^{N}\left|e_{i}^{n}\right|\left|\nabla_{h}^{-} e_{i}^{n+1}\right|+\left|e_{i-1}^{n}\right|\left|\nabla_{h}^{-} e_{i}^{n+1}\right|\right) \\
& \leq 6 s c_{2} \Delta t\left[2\left(\Delta x \sum_{i=1}^{N}\left(e_{i}^{n}\right)^{2}\right)^{1 / 2}\left(\Delta x \sum_{i=1}^{N}\left(\nabla_{h}^{-} e_{i}^{n+1}\right)^{2}\right)^{1 / 2}\right] \\
& \leq 12 s c_{2} \Delta t\left\|\mathbf{e}^{n}\right\|_{h}\left|\mathbf{e}^{n+1}\right|_{1, h} \\
& \leq 6 \Delta t\left(\frac{s^{2} c_{2}^{2}}{\delta_{1}}\left\|\mathbf{e}^{n}\right\|_{h}^{2}+\delta_{1}\left|\mathbf{e}^{n+1}\right|_{1, h}^{2}\right)
\end{aligned}
$$




$$
\begin{aligned}
3 \Delta t \Delta x & \sum_{i=1}^{N}\left[\left(e_{i}^{n}\right)^{2}+\left(e_{i-1}^{n}\right)^{2}\right] \nabla_{h}^{-} v_{i}^{n+1} \nabla_{h}^{-} e_{i}^{n+1} \\
& \leq 3 c_{2} \Delta t\left(\Delta x \sum_{i=1}^{N}\left[\left(e_{i}^{n}\right)^{2}+\left(e_{i-1}^{n}\right)^{2}\right]\left|\nabla_{h}^{-} e_{i}^{n+1}\right|\right) \\
& \leq 3 c_{2} \Delta t\left\|\mathbf{e}^{n}\right\|_{\infty}\left(\Delta x \sum_{i=1}^{N}\left[\left|e_{i}^{n}\right|+\left|e_{i-1}^{n}\right|\right]\left|\nabla_{h}^{-} e_{i}^{n+1}\right|\right) \\
& \leq 6 c_{2} \Delta t\left\|\mathbf{e}^{n}\right\|_{\infty, h}\left[\left(\Delta x \sum_{i=1}^{N}\left(e_{i}^{n}\right)^{2}\right)^{1 / 2}\left(\Delta x \sum_{i=1}^{N}\left(\nabla_{h}^{-} e_{i}^{n+1}\right)^{2}\right)^{1 / 2}\right] \\
& =6 c_{2} \Delta t\left\|\mathbf{e}^{n}\right\|_{\infty, h}\left\|\mathbf{e}^{n}\right\|_{h}\left|\mathbf{e}^{n+1}\right|_{1, h} \\
& \leq 3 \Delta t\left(\frac{c_{2}^{2}}{\delta_{2}}\left\|\mathbf{e}^{n}\right\|_{h}^{2}+\delta_{2}\left\|\mathbf{e}^{n}\right\|_{\infty, h}^{2}\left|\mathbf{e}^{n+1}\right|_{1, h}^{2}\right) .
\end{aligned}
$$

From the definition of the bilinear map $C_{h}$, we have

$$
\begin{aligned}
\left(C_{h}\left(\boldsymbol{v}^{n+1}, \tilde{\mathbf{e}}^{n}\right), \mathbf{e}^{n+1}\right)_{h}= & \Delta x \alpha \sum_{i=1}^{N}\left[v_{i}^{n+1}\left(\nabla_{h}^{+}\right) \tilde{e}_{i}^{n}+\tilde{e}_{i}^{n}\left(\nabla_{h}^{-}\right) v_{i}^{n+1}+\tilde{e}_{i+1}^{n} \nabla_{h}^{+} v_{i}^{n+1}\right] e_{i}^{n+1} \\
& +\Delta x \alpha \sum_{i=1}^{N}\left[v_{i}^{n+1}\left(\nabla_{h}^{-}\right) \tilde{e}_{i}^{n}+\tilde{e}_{i}^{n}\left(\nabla_{h}^{+}\right) v_{i}^{n+1}+\tilde{e}_{i-1}^{n} \nabla_{h}^{-} v_{i}^{n+1}\right] e_{i}^{n+1} .
\end{aligned}
$$

We now estimate each of the terms in (3.24).

$$
\begin{aligned}
\Delta x \sum_{i=1}^{N} v_{i}^{n+1}\left(\nabla_{h}^{-} \tilde{e}_{i}^{n}\right) e_{i}^{n+1} & =-\Delta x \sum_{i=1}^{N} \tilde{e}_{i}^{n}\left(\nabla_{h}^{+}\left(v_{i}^{n+1} e_{i}^{n+1}\right)\right) \\
= & -\Delta x \sum_{i=1}^{N} \tilde{e}_{i}^{n}\left[\left(\nabla_{h}^{+} v_{i}^{n+1}\right) e_{i+1}^{n+1}+v_{i}^{n+1} \nabla_{h}^{+} e_{i}^{n+1}\right] \\
\leq & c_{2}\left\|\tilde{\mathbf{e}}^{n}\right\|_{h}\left\|\mathbf{e}^{n+1}\right\|_{h}+s\left\|\tilde{\mathbf{e}}^{n}\right\|_{h}\left|\mathbf{e}^{n+1}\right|_{1, h} . \\
\Delta x \sum_{i=1}^{N} \tilde{e}_{i}^{n}\left(\nabla_{h}^{-} v_{i}^{n+1}\right) e_{i}^{n+1} & \leq \max _{1 \leq i \leq N}\left|\left(\nabla_{h}^{-}\right) v_{i}^{n+1}\right|\left[\Delta x \Delta y \sum_{i=1}^{N}\left|\tilde{e}_{i}^{n}\right|\left|e_{i}^{n+1}\right|\right] \\
& \leq c_{2}\left\|\tilde{\mathbf{e}}^{n}\right\|\left\|_{h}\right\| \mathbf{e}^{n+1} \|_{h} . \\
\Delta x \sum_{i=1}^{N}\left[\tilde{e}_{i+1}^{n} \nabla_{h}^{+} v_{i}^{n+1}\right] e_{i}^{n+1} & \leq \max _{1 \leq i \leq N}\left|\nabla_{h}^{+} v_{i}^{n+1}\right|\left[\Delta x \sum_{i=1}^{N}\left|\tilde{e}_{i+1}^{n}\right|\left|e_{i}^{n+1}\right|\right] \\
& \leq c_{2}\left\|\tilde{\mathbf{e}}^{n}\right\|_{h}\left\|\mathbf{e}^{n+1}\right\|_{h} .
\end{aligned}
$$


In a similar fashion, we obtain

$$
\begin{aligned}
& \Delta x \sum_{i=1}^{N} v_{i, j}^{n+1}\left(\nabla_{h}^{-} \tilde{e}_{i}^{n}\right) e_{i}^{n+1} \leq c_{2}\left\|\tilde{\mathbf{e}}^{n}\right\|_{h}\left\|\mathbf{e}^{n+1}\right\|_{h}+s\left\|\tilde{\mathbf{e}}^{n}\right\|_{h}\left|\mathbf{e}^{n+1}\right|_{1, h} . \\
& \Delta x \sum_{i=1}^{N} \tilde{e}_{i}^{n}\left(\nabla_{h}^{+} v_{i}^{n+1}\right) e_{i}^{n+1} \leq c_{2}\left\|\tilde{\mathbf{e}}^{n}\right\|_{h}\left\|\mathbf{e}^{n+1}\right\|_{h} . \\
& \Delta x \sum_{i=1}^{N}\left[\tilde{e}_{i-1}^{n} \nabla_{h}^{-} v_{i}^{n+1}\right] e_{i}^{n+1} \leq c_{2}\left\|\tilde{\mathbf{e}}^{n}\right\|_{h}\left\|\mathbf{e}^{n+1}\right\|_{h} .
\end{aligned}
$$

Combining the inequalities from (3.25)-(3.30), we have

$$
\begin{aligned}
2 \Delta t\left(C_{h}\left(\boldsymbol{v}^{n+1}, \tilde{\mathbf{e}}^{n}\right), \mathbf{e}^{n+1}\right)_{h} & \leq 2 \Delta t|\alpha|\left[2 s\left\|\tilde{\mathbf{e}}^{n}\right\|_{h}\left|\mathbf{e}^{n+1}\right|_{1, h}+6 c_{2}\left\|\tilde{\mathbf{e}}^{n}\right\|_{h}\left\|\mathbf{e}^{n+1}\right\|_{h}\right] \\
& \leq \Delta t\left[\frac{4 s^{2} \alpha^{2}}{\delta_{4}}\left\|\tilde{\mathbf{e}}^{n}\right\|_{h}^{2}+\delta_{4}\left|\mathbf{e}^{n+1}\right|_{1, h}^{2}\right]+\Delta t\left[\frac{36 c_{2}^{2} \alpha^{2}}{\delta_{5}}\left\|\tilde{\mathbf{e}}^{n}\right\|_{h}^{2}+\delta_{5}\left\|\mathbf{e}^{n+1}\right\|_{h}^{2}\right]
\end{aligned}
$$

The last term of (3.20) is estimated as follows.

$$
\begin{aligned}
\left(\mathbf{r}^{n}, \mathbf{e}^{n+1}\right)_{h} & \leq \Delta x \sum_{i=1}^{N} r_{i}^{n} e_{i}^{n+1} \\
& \leq N \Delta x \max _{1 \leq i \leq N}\left|r_{i}^{n}\right|\left(\Delta x \sum_{i=1}^{N}\left(e_{i}^{n+1}\right)^{2}\right)^{1 / 2} \\
& \leq \frac{4 L^{2}}{2 \delta_{3}}\left(\max _{1 \leq i \leq N}\left|r_{i}^{n}\right|\right)^{2}+\frac{\delta_{3}}{2}\left\|\mathbf{e}^{n+1}\right\|_{h}^{2} \\
& \leq \frac{4 L^{2} c_{1}^{2}}{2 \delta_{3}}\left(\Delta t+\Delta x^{2}\right)^{2}+\frac{\delta_{3}}{2}\left\|\mathbf{e}^{n+1}\right\|_{h}^{2}
\end{aligned}
$$

Thus, from (3.22), (3.23), (3.31) and (3.32), we get

$$
\begin{aligned}
\left\|\mathbf{e}^{n+1}\right\|_{h}^{2}- & \left\|\mathbf{e}^{n}\right\|_{h}^{2}+2 \Delta t \varepsilon^{2}\left|\mathbf{e}^{n+1}\right|_{2, h}^{2} \leq 2 \Delta t\left|\mathbf{e}^{n+1}\right|_{1, h}^{2}+6 \Delta t\left(\frac{s^{2} c_{2}^{2}}{\delta_{1}}\left\|\mathbf{e}^{n}\right\|_{h}^{2}+\delta_{1}\left|\mathbf{e}^{n+1}\right|_{1, h}^{2}\right) \\
& +3 \Delta t\left(\frac{c_{2}^{2}}{\delta_{2}}\left\|\mathbf{e}^{n}\right\|_{h}^{2}+\delta_{2}\left\|\mathbf{e}^{n}\right\|_{\infty, h}^{2}\left|\mathbf{e}^{n+1}\right|_{1, h}^{2}\right)+\Delta t\left[\frac{4 s^{2} \alpha^{2}}{\delta_{4}}\left\|\tilde{\mathbf{e}}^{n}\right\|_{h}^{2}+\delta_{4}\left|\mathbf{e}^{n+1}\right|_{1, h}^{2}\right] \\
& +\Delta t\left[\frac{36 c_{2}^{2} \alpha^{2}}{\delta_{5}}\left\|\tilde{\mathbf{e}}^{n}\right\|_{h}^{2}+\delta_{5}\left\|\mathbf{e}^{n+1}\right\|_{h}^{2}\right]+\frac{4 \Delta t L^{2} c_{1}^{2}}{\delta_{3}}\left(\Delta t+\Delta x^{2}\right)^{2}+\Delta t \delta_{3}\left\|\mathbf{e}^{n+1}\right\|_{h}^{2},
\end{aligned}
$$

which on rearrangement gives

$$
\begin{aligned}
{\left[1-\Delta t\left(\delta_{3}+\delta_{5}\right)\right]\left\|\mathbf{e}^{n+1}\right\|_{h}^{2}+2 \Delta t \varepsilon^{2}\left|\mathbf{e}^{n+1}\right|_{2, h}^{2} } & \leq \Delta t\left[2+6 \delta_{1}+\delta_{4}\right]\left|\mathbf{e}^{n+1}\right|_{1, h}^{2}+\left[1+6 \Delta t c_{2}^{2}\left(\frac{s^{2}}{\delta_{1}}+\frac{1}{\delta_{2}}\right)\right]\left\|\mathbf{e}^{n}\right\|_{h}^{2} \\
& +3 \Delta t \delta_{2}\left\|\mathbf{e}^{n}\right\|_{\infty, h}^{2}\left|\mathbf{e}^{n+1}\right|_{1, h}^{2}+4 \Delta t \alpha^{2}\left[\frac{s^{2}}{\delta_{4}}+\frac{9 c_{2}^{2}}{\delta_{5}}\right]\left\|\tilde{\mathbf{e}}^{n}\right\|_{h}^{2} \\
& +\frac{4 \Delta t L^{2} c_{1}^{2}}{\delta_{3}}\left(\Delta t+\Delta x^{2}\right)^{2}
\end{aligned}
$$

Using (2.4), Lemma 2.2 and Young's inequality, (3.33) becomes 


$$
\begin{aligned}
{\left[1-\Delta t\left(\delta_{3}+\delta_{5}\right)\right]\left\|\mathbf{e}^{n+1}\right\|_{h}^{2}+2 \Delta t \varepsilon^{2}\left|\mathbf{e}^{n+1}\right|_{2, h}^{2} } & \leq \frac{\Delta t\left(2+6 \delta_{1}+\delta_{4}\right)^{2}}{4 \varepsilon^{2}}\left\|\mathbf{e}^{n+1}\right\|_{h}^{2}+\Delta t \varepsilon^{2}\left|\mathbf{e}^{n+1}\right|_{2, h}^{2} \\
& +\left[1+6 \Delta t\left(\frac{s^{2} c_{2}^{2}}{\delta_{1}}+\frac{c_{2}^{2}}{\delta_{2}}\right)\right]\left\|\mathbf{e}^{n}\right\|_{h}^{2} \\
& +\frac{3 \Delta t \delta_{2}}{\Delta x}\left\|\mathbf{e}^{n}\right\|_{h}^{2}\left|\mathbf{e}^{n+1}\right|_{1, h}^{2}+4 \Delta t \alpha^{2}\left[\frac{s^{2}}{\delta_{4}}+\frac{9 c_{2}^{2}}{\delta_{5}}\right]\left\|\tilde{\mathbf{e}}^{n}\right\|_{h}^{2} \\
& +\frac{4 \Delta t L^{2} c_{1}^{2}}{\delta_{3}}\left(\Delta t+\Delta x^{2}\right)^{2} .
\end{aligned}
$$

Using (2.14), (3.34) gives

$$
\begin{gathered}
{[1-\Delta t c]\left\|\mathbf{e}^{n+1}\right\|_{h}^{2}+\Delta t \varepsilon^{2} \eta^{2}\left|\mathbf{e}^{n+1}\right|_{1, h}^{2} \leq\left(1+\Delta t c_{5}\right)\left\|\mathbf{e}^{n}\right\|_{h}^{2}+\Delta t c_{4}\left\|\tilde{\mathbf{e}}^{n}\right\|_{h}^{2}+\frac{3 \Delta t \delta_{2}}{\Delta x}\left\|\mathbf{e}^{n}\right\|_{h}^{2}\left|\mathbf{e}^{n+1}\right|_{1, h}^{2}} \\
+\Delta t c_{3}\left(\Delta t+\Delta x^{2}\right)^{2}
\end{gathered}
$$

where

$$
\begin{aligned}
& c_{3}=\frac{4 L^{2} c_{1}^{2}}{\delta_{3}}, \quad c=\delta_{3}+\delta_{5}+\frac{\left(2+6 \delta_{1}+\delta_{4}\right)^{2}}{4 \varepsilon^{2}} \\
& c_{4}=6\left(\frac{s^{2} c_{2}^{2}}{\delta_{1}}+\frac{c_{2}^{2}}{\delta_{2}}\right), \quad c_{5}=4 \alpha^{2}\left[\frac{s^{2}}{\delta_{4}}+\frac{9 c_{2}^{2}}{\delta_{5}}\right] .
\end{aligned}
$$

For

$$
\Delta t \leq \frac{1}{2 c} \equiv c
$$

then it follows from (3.35) that

$$
\begin{gathered}
\left\|\mathbf{e}^{n+1}\right\|_{h}^{2}+\Delta t \varepsilon^{2} \eta^{2}\left|\mathbf{e}^{n+1}\right|_{1, h}^{2} \leq 4^{\Delta t c}\left[\left(1+\Delta t c_{5}\right)\left\|\mathbf{e}^{n}\right\|_{h}^{2}+\Delta t c_{4}\left\|\tilde{\mathbf{e}}^{n}\right\|_{h}^{2}+\frac{3 \Delta t \delta_{2}}{\Delta x}\left\|\mathbf{e}^{n}\right\|_{h}^{2}\left|\mathbf{e}^{n+1}\right|_{1, h}^{2}\right. \\
\left.+\Delta t c_{3}\left(\Delta t+\Delta x^{2}\right)^{2}\right]
\end{gathered}
$$

Now for

$$
\frac{3 \delta_{2} c_{3}}{\Delta x}\left(\Delta t+\Delta x^{2}\right)^{2} \leq \frac{c_{6}}{2} \varepsilon^{2} \eta^{2} 4^{-T c} \exp \left(-T c_{6}\right),
$$

where $c_{6}=c_{5}+m_{0} A c_{4}$ and $A=\sum_{i=1}^{m_{0}}\left|a_{i}\right|^{2}$, we prove by inductive method that

$$
\left\|\mathbf{e}^{n+1}\right\|_{h}^{2}+\frac{1}{2} \Delta t \varepsilon^{2} \eta^{2}\left|\mathbf{e}^{n+1}\right|_{1, h}^{2} \leq 4^{\Delta t c}\left[\left(1+\Delta t c_{5}\right)\left\|\mathbf{e}^{n}\right\|_{h}^{2}+\Delta t c_{4}\left\|\tilde{\mathbf{e}}^{n}\right\|_{h}^{2}+\Delta t c_{3}\left(\Delta t+\Delta x^{2}\right)^{2}\right] .
$$

For $n=0$ from (3.37), one obtains

$$
\begin{aligned}
\left\|\mathbf{e}^{1}\right\|_{h}^{2}+\Delta t \varepsilon^{2} \eta^{2}\left|\mathbf{e}^{1}\right|_{1, h}^{2} \leq & 4^{\Delta t c}\left[\left(1+\Delta t\left(c_{5}+c_{4}\right)\right)\left\|\mathbf{e}^{0}\right\|_{h}^{2}+\frac{3 \Delta t \delta_{2}}{\Delta x}\left\|\mathbf{e}^{0}\right\|_{h}^{2}\left|\mathbf{e}^{1}\right|_{1, h}^{2}\right] \\
& +\Delta t c_{3}\left(4^{\Delta t c}\right)\left[\left(\Delta t+\Delta x^{2}\right)^{2}\right]
\end{aligned}
$$

and hence,

$$
\left\|\mathbf{e}^{1}\right\|_{h}^{2}+\frac{1}{2} \Delta t \varepsilon^{2} \eta^{2}\left|\mathbf{e}^{1}\right|_{1, h}^{2} \leq 4^{\Delta t c}\left[\left(1+\Delta t\left(c_{5}+c_{4}\right)\right)\left\|\mathbf{e}^{0}\right\|_{h}^{2}+\Delta t c_{3}\left(\Delta t+\Delta x^{2}\right)^{2}\right],
$$


which is (3.39) for $n=0$. Now suppose that (3.39) is true up to the order $n-1$. Thus, for $s=0,1, \ldots, n-1$,

$$
\left\|\mathbf{e}^{s+1}\right\|_{h}^{2}+\frac{1}{2} \Delta t \varepsilon^{2} \eta^{2}\left|\mathbf{e}^{s+1}\right|_{1, h}^{2} \leq 4^{\Delta t c}\left[\left(1+\Delta t c_{5}\right)\left\|\mathbf{e}^{s}\right\|_{h}^{2}+\Delta t c_{4}\left\|\tilde{\mathbf{e}}^{s}\right\|_{h}^{2}+\Delta t c_{3}\left(\Delta t+\Delta x^{2}\right)^{2}\right] .
$$

It is now remaining to treat the term $\left\|\tilde{\mathbf{e}}^{s}\right\|_{h}^{2}$. For $s<m_{0}-1$, it is clear from (3.4) that $\left\|\tilde{\mathbf{e}}^{s}\right\|_{h}=\left\|\mathbf{e}^{s}\right\|_{h}$. Thus, (3.40) becomes

$$
\left\|\mathbf{e}^{s+1}\right\|_{h}^{2}+\frac{1}{2} \Delta t \varepsilon^{2} \eta^{2}\left|\mathbf{e}^{s+1}\right|_{1, h}^{2} \leq 4^{\Delta t c}\left[\left(1+\Delta t\left(c_{5}+c_{4}\right)\right)\left\|\mathbf{e}^{s}\right\|_{h}^{2}+\Delta t c_{3}\left(\Delta t+\Delta x^{2}\right)^{2}\right] .
$$

It follows from (3.41) that

$$
\left\|\mathbf{e}^{s+1}\right\|_{h}^{2} \leq 4^{\Delta t c}\left[\left(1+\Delta t\left(c_{5}+c_{4}\right)\right)\left\|\mathbf{e}^{s}\right\|_{h}^{2}+\Delta t c_{3}\left(\Delta t+\Delta x^{2}\right)^{2}\right] .
$$

After $s+1$ iterations, we get

$$
\begin{aligned}
\left\|\mathbf{e}^{s+1}\right\|_{h}^{2} & \leq 4^{\Delta t(s+1) c}\left[\left(1+\Delta t\left(c_{5}+c_{4}\right)\right)^{s+1}\left\|\mathbf{e}^{0}\right\|_{h}^{2}+\Delta t c_{3}\left(\Delta t+\Delta x^{2}\right)^{2} \sum_{j=0}^{s}\left(1+\Delta t\left(c_{5}+c_{4}\right)\right)^{j}\right] \\
& =4^{\Delta t(s+1) c}\left[\Delta t c_{3}\left(\Delta t+\Delta x^{2}\right)^{2} \sum_{j=0}^{s}\left(1+\Delta t\left(c_{5}+c_{4}\right)\right)^{j}\right] .
\end{aligned}
$$

For the case $s \geq m_{0}-1$ and $m_{0}>1$, it follows from (3.2) that

$$
\left\|\tilde{\mathbf{e}}^{s}\right\|_{h} \leq \sum_{i=1}^{m_{0}}\left|a_{i}\right|\left\|\tilde{\mathbf{e}}^{s-i+1}\right\|_{h}
$$

which by Cauchy-Schwartz's inequality gives

$$
\left\|\tilde{\mathbf{e}}^{s}\right\|_{h}^{2} \leq A\left[\left\|\mathbf{e}^{s}\right\|_{h}^{2}+\left\|\mathbf{e}^{s-1}\right\|_{h}^{2}+\cdots+\left\|\mathbf{e}^{s-m_{0}+1}\right\|_{h}^{2}\right] .
$$

Hence, (3.40) gives

$$
\begin{aligned}
\left\|\mathbf{e}^{s+1}\right\|_{h}^{2} & \leq 4^{\Delta t c}\left[\left(1+\Delta t c_{5}\right)\left\|\mathbf{e}^{s}\right\|_{h}^{2}+\Delta t c_{4} A\left(\left\|\mathbf{e}^{s}\right\|_{h}^{2}+\left\|\mathbf{e}^{s-1}\right\|_{h}^{2}+\cdots+\left\|\mathbf{e}^{s-m_{0}+1}\right\|_{h}^{2}\right)\right] \\
& +4^{\Delta t c}\left[\Delta t c_{3}\left(\Delta t+\Delta x^{2}\right)^{2}\right] .
\end{aligned}
$$

It follows easily that

$$
\begin{aligned}
\max \left\{\left\|\mathbf{e}^{s+1}\right\|_{h}^{2},\left\|\mathbf{e}^{s}\right\|_{h}^{2}, \ldots,\right. & \left.\left\|\mathbf{e}^{s-m_{0}+2}\right\|_{h}^{2}\right\} \leq 4^{\Delta t c}\left[\left(1+\Delta t c_{6}\right) \max \left\{\left\|\mathbf{e}^{s}\right\|_{h}^{2},\left\|\mathbf{e}^{s-1}\right\|_{h}^{2}, \ldots,\left\|\mathbf{e}^{s-m_{0}+1}\right\|_{h}^{2}\right\}\right. \\
& \left.+\Delta t c_{3}\left(\Delta t+\Delta x^{2}\right)^{2}\right]
\end{aligned}
$$

which after $s-m_{0}+2$ iterations gives

$$
\begin{aligned}
& \max \left\{\left\|\mathbf{e}^{s+1}\right\|_{h}^{2},\left\|\mathbf{e}^{s}\right\|_{h}^{2}, \ldots,\right.\left.\left\|\mathbf{e}^{s-m_{0}+2}\right\|_{h}^{2}\right\} \\
& \leq 4^{\left(s-m_{0}+2\right) \Delta t c}\left[\left(1+\Delta t c_{6}\right)^{s-m_{0}+2} \max \left\{\left\|\mathbf{e}^{m_{0}-1}\right\|_{h}^{2},\left\|\mathbf{e}^{m_{0}-2}\right\|_{h}^{2}, \ldots,\left\|\mathbf{e}^{0}\right\|_{h}^{2}\right\}\right. \\
&\left.\quad+\Delta t c_{3}\left(\Delta t+\Delta x^{2}\right)^{2} \sum_{j=0}^{s-m_{0}+1}\left(1+\Delta t c_{6}\right)^{j}\right] .
\end{aligned}
$$


Combining (3.42) and (3.46), we get

$$
\begin{aligned}
\left\|\mathbf{e}^{s+1}\right\|_{h}^{2} & \leq 4^{(s+1) \Delta t c}\left[\Delta t c_{3}\left(\Delta t+\Delta x^{2}\right)^{2} \sum_{j=0}^{s}\left(1+\Delta t c_{6}\right)^{j}\right] \\
& =4^{(s+1) \Delta t c}\left[\Delta t c_{3}\left(\Delta t+\Delta x^{2}\right)^{2}\left(\frac{\left(1+\Delta t c_{6}\right)^{s+1}-1}{\Delta t c_{6}}\right)\right] \\
& \leq 4^{(s+1) \Delta t c} \exp \left((s+1) \Delta t c_{6}\right)\left[\frac{c_{3}}{c_{6}}\left(\Delta t+\Delta x^{2}\right)^{2}\right]
\end{aligned}
$$

and

$$
\left\|\tilde{\mathbf{e}}^{s+1}\right\|_{h}^{2} \leq m_{0} A 4^{(s+1) \Delta t c} \exp \left((s+1) \Delta t c_{6}\right)\left[\frac{c_{3}}{c_{6}}\left(\Delta t+\Delta x^{2}\right)^{2}\right]
$$

for $s=0,1, \ldots, n-1$.

Going back to (3.37), we have

$$
\begin{gathered}
\left\|\mathbf{e}^{n+1}\right\|_{h}^{2}+\Delta t \varepsilon^{2} \eta^{2}\left|\mathbf{e}^{n+1}\right|_{1, h}^{2} \leq 4^{\Delta t c}\left[\left(1+\Delta t c_{5}\right)\left\|\mathbf{e}^{n}\right\|_{h}^{2}+\Delta t c_{4}\left\|\tilde{\mathbf{e}}^{n}\right\|_{h}^{2}+\frac{3 \Delta t \delta_{2}}{\Delta x}\left\|\mathbf{e}^{n}\right\|_{h}^{2}\left|\mathbf{e}^{n+1}\right|_{1, h}^{2}\right] \\
+\Delta t c_{3} 4^{\Delta t c}\left(\Delta t+\Delta x^{2}\right)^{2} \\
\leq 4^{\Delta t c}\left[\left(1+\Delta t c_{5}\right)\left\|\mathbf{e}^{n}\right\|_{h}^{2}+\Delta t c_{4}\left|\tilde{\mathbf{e}}^{n}\right|_{h}^{2}+\Delta t c_{3}\left(\Delta t+\Delta x^{2}\right)^{2}\right] \\
+\frac{3 \Delta t \delta_{2}}{\Delta x} 4^{n \Delta t c} \exp \left(n \Delta t c_{6}\right)\left[\frac{c_{3}}{c_{6}}\left(\Delta t+\Delta x^{2}\right)^{2}\right]\left|\mathbf{e}^{n+1}\right|_{1, h}^{2}
\end{gathered}
$$

which by (3.38) gives

$$
\left\|\mathbf{e}^{n+1}\right\|_{h}^{2}+\Delta t \varepsilon^{2} \eta^{2}\left|\mathbf{e}^{n+1}\right|_{1, h}^{2} \leq 4^{\Delta t c}\left[\left(1+\Delta t c_{5}\right)\left\|\mathbf{e}^{n}\right\|_{h}^{2}+\Delta t c_{4}\left\|\tilde{\mathbf{e}}^{n}\right\|_{h}^{2}+\Delta t c_{3}\left(\Delta t+\Delta x^{2}\right)^{2}\right]+\frac{1}{2} \Delta t \varepsilon^{2} \eta^{2}\left|\mathbf{e}^{n+1}\right|_{1, h}^{2} .
$$

Thus, we obtain

$$
\left\|\mathbf{e}^{n+1}\right\|_{h}^{2}+\frac{1}{2} \Delta t \varepsilon^{2} \eta^{2}\left|\mathbf{e}^{n+1}\right|_{1, h}^{2} \leq 4^{\Delta t c}\left[\left(1+\Delta t c_{5}\right)\left\|\mathbf{e}^{n}\right\|_{h}^{2}+\Delta t c_{4}\left\|\tilde{\mathbf{e}}^{n}\right\|_{h}^{2}+\Delta t c_{3}\left(\Delta t+\Delta x^{2}\right)^{2}\right] .
$$

Using (3.47) and (3.48), (3.49) gives

$$
\begin{aligned}
\left\|\mathbf{e}^{n}\right\|_{h}^{2} & \leq 4^{n \Delta t c} \exp \left(n \Delta t c_{6}\right)\left[\frac{c_{3}}{c_{6}}\left(\Delta t+\Delta x^{2}\right)^{2}\right] \\
& \leq 4^{T c} \exp \left(T c_{6}\right)\left[\frac{c_{3}}{c_{6}}\left(\Delta t+\Delta x^{2}\right)^{2}\right],
\end{aligned}
$$

for $n=1,2, \ldots, M$. Thus, it follows from (3.50) that

$$
\left\|\mathbf{e}^{n}\right\|_{h} \leq C\left(\Delta t+\Delta x^{2}\right),
$$

for a constant $C$ independent of $\Delta t$ and $\Delta x$. This completes the proof.

Remark 3.2. The condition $\Delta t<4 \varepsilon^{2}$ in the convergence analysis expresses that one needs the solution of the discrete system (3.6a)-(3.6c) to exists. It is worth noting that the convergence result Theorem 3.3 is conducted for $\alpha_{1}=\alpha_{2}$. The case when $\alpha_{1} \neq \alpha_{2}$ is treated in the same way but the rate of convergence will change. 


\subsection{Explicit finite volume method}

Here, we approximate the solution of Eq. (1.1) using an explicit finite volume method:

$$
\left\{\begin{array}{l}
\frac{u_{i}^{n+1}-u_{i}^{n}}{\Delta t}-\left(C_{h}\left(\mathbf{u}^{n}, \mathbf{u}^{n}\right)\right)_{i}+\varepsilon^{2} \Delta_{h}^{2} u_{i}^{n}=\nabla_{h}^{+}\left[\varphi_{i-\frac{1}{2}}^{n} \nabla_{h}^{-} u_{i}^{n}\right] . \\
u_{i}^{n}=u_{i+N}^{n} \\
u_{i}^{0}=\frac{1}{\Delta x} \int_{k_{i}} u^{0}(x) d x
\end{array}\right.
$$

for $1 \leq n \leq M-1$ and $1 \leq i \leq N$.

The finite volume discretization (3.51) is explicit, hence the solution $\mathbf{u}^{n}$ is computed at each time step, but one of important feature of this scheme is the following:

Theorem 3.4. We assume that the following are satisfied for some $\delta, 0<\delta<1$ :

$$
\begin{aligned}
& \frac{\Delta t}{\Delta x^{4}} \leq \frac{1-\delta}{64 \varepsilon^{2}} \\
& \frac{16 \Delta t}{\Delta x^{2}} \leq \varepsilon^{2} \delta \eta^{2}(1-\delta) \\
& \frac{72 \Delta t}{\Delta x}\left(\left(\left|\alpha_{1}\right|+\left|\alpha_{2}\right|\right)^{2}+\frac{4}{\Delta x^{3}}\left\|\boldsymbol{u}^{0}\right\|_{h}^{2}\right)\left\|\boldsymbol{u}^{0}\right\|_{h}^{2} \leq \varepsilon^{2} \delta^{2} \eta^{2} \exp \left(\frac{-2 T}{\varepsilon^{2}}\right) .
\end{aligned}
$$

Then the finite volume method defined by (3.51) is stable is $L^{\infty}\left(0, T ; \mathcal{H}_{h}\right)$ stable in the following sense:

$$
\begin{aligned}
& \left\|\boldsymbol{u}^{n}\right\|_{h}^{2} \leq \exp \left(\frac{\Delta t}{\varepsilon^{2}}\right)\left\|\boldsymbol{u}^{n-1}\right\|_{h}^{2} \leq \cdots \leq \exp \left(\frac{n \Delta t}{\varepsilon^{2}}\right)\left\|\boldsymbol{u}^{0}\right\|_{h}^{2} \leq \exp \left(\frac{T}{\varepsilon^{2}}\right)\left\|\boldsymbol{u}^{0}\right\|_{h}^{2}, n=1,2 \ldots, M, \\
& \frac{\Delta t}{2} \varepsilon^{2} \delta^{2} \eta^{2} \sum_{n=0}^{M-1} \exp \left(\frac{(M-n) \Delta t}{\varepsilon^{2}}\right)\left|\boldsymbol{u}^{n}\right|_{1, h}^{2} \leq \exp \left(\frac{T}{\varepsilon^{2}}\right)\left\|\boldsymbol{u}^{0}\right\|_{h}^{2}
\end{aligned}
$$

Proof. To prove the stability we use the approach used in [15]. Multiplying Eq. (3.51) by $2 \Delta t \Delta x u_{i}^{n}$ and summing the equalities for $i=1, \ldots, N$ together with (2.6) and Lemma 2.2, we arrive at

$$
\left\|\mathbf{u}^{n+1}\right\|_{h}^{2}-\left\|\mathbf{u}^{n+1}-\mathbf{u}^{n}\right\|_{h}^{2}+\Delta t \varepsilon^{2}\left|\mathbf{u}^{n}\right|_{2, h}^{2} \leq \exp \left(\frac{\Delta t}{\varepsilon^{2}}\right)\left\|\mathbf{u}^{n}\right\|_{h}^{2} .
$$

Now we have to estimate the term $\left\|\mathbf{u}^{n+1}-\mathbf{u}^{n}\right\|_{h}^{2}$. By multiplying Eq. (3.51) by $2 \Delta t \Delta x\left(u_{i}^{n+1}-u_{i}^{n}\right)$ and adding the corresponding equalities for $i=1, \ldots, N$, we obtain

$$
\begin{aligned}
2\left\|\mathbf{u}^{n+1}-\mathbf{u}^{n}\right\|_{h}^{2} & -2 \Delta t\left(C_{h}\left(\mathbf{u}^{n}, \mathbf{u}^{n}\right), \mathbf{u}^{n+1}-\mathbf{u}^{n}\right)_{h}+2 \Delta t \varepsilon^{2}\left(\Delta_{h} \mathbf{u}^{n}, \Delta_{h}\left(\mathbf{u}^{n+1}-\mathbf{u}^{n}\right)\right) \\
& =-2 \Delta t \Delta x \sum_{i=1}^{N}\left[\varphi_{i-\frac{1}{2}}^{n} \nabla_{h}^{-} u_{i}^{n} \nabla_{h}^{-}\left(u_{i}^{n+1}-u_{i}^{n}\right)\right] \\
& =-2 \Delta t \Delta x \sum_{i=1}^{N}\left[\left(\varphi_{i-\frac{1}{2}}^{n}+1\right) \nabla_{h}^{-} u_{i}^{n} \nabla_{h}^{-}\left(u_{i}^{n+1}-u_{i}^{n}\right)\right]+2 \Delta t\left(\nabla_{h}^{-} \mathbf{u}^{n}, \nabla_{h}^{-}\left(\mathbf{u}^{n+1}-\mathbf{u}^{n}\right)\right)_{h} .
\end{aligned}
$$

Using (2.8) and (2.9), we majorize all the terms in (4.26) as follows:

$$
\begin{aligned}
2 \Delta t\left(C_{h}\left(\mathbf{u}^{n}, \mathbf{u}^{n}\right), \mathbf{u}^{n+1}-\mathbf{u}^{n}\right)_{h} & \leq \frac{36 \Delta t^{2}}{\Delta x}\left(\left|\alpha_{1}\right|+\left|\alpha_{2}\right|\right)^{2}\left\|\mathbf{u}^{n}\right\|_{h}^{2}\left|\mathbf{u}^{n}\right|_{1, h}^{2}+\frac{1}{4}\left\|\mathbf{u}^{n+1}-\mathbf{u}^{n}\right\|_{h}^{2} ; \\
-2 \Delta t \varepsilon^{2}\left(\Delta_{h} \mathbf{u}^{n}, \Delta_{h}\left(\mathbf{u}^{n+1}-\mathbf{u}^{n}\right)\right)_{h} & \leq \frac{64 \varepsilon^{4} \Delta t^{2}}{\Delta x^{4}}\left|\mathbf{u}^{n}\right|_{2, h}^{2}+\frac{1}{4}\left\|\mathbf{u}^{n+1}-\mathbf{u}^{n}\right\|_{h}^{2} ; \\
-2 \Delta t \Delta x \sum_{i=1}^{N}\left[\left(\varphi_{i-\frac{1}{2}}^{n}+1\right) \nabla_{h}^{-} u_{i}^{n} \nabla_{h}^{-}\left(u_{i}^{n+1}-u_{i}^{n}\right)\right] & \leq 144 \frac{\Delta t^{2}}{\Delta x^{4}}\left\|\mathbf{u}^{n}\right\|_{h}^{4}\left|\mathbf{u}^{n}\right|_{1, h}^{2}+\frac{1}{4}\left\|\mathbf{u}^{n+1}-\mathbf{u}^{n}\right\|_{h}^{2} ; \\
2 \Delta t\left(\nabla_{h}^{-} \mathbf{u}^{n}, \nabla_{h}^{-}\left(\mathbf{u}^{n+1}-\mathbf{u}^{n}\right)\right)_{h} & \leq \frac{16 k^{2}}{\Delta x^{2}}\left|\mathbf{u}^{n}\right|_{1, h}^{2}+\frac{1}{4}\left\|\mathbf{u}^{n+1}-\mathbf{u}^{n}\right\|_{h}^{2} .
\end{aligned}
$$


Thus using (3.57), (3.56) becomes

$$
\begin{aligned}
\left\|\mathbf{u}^{n+1}-\mathbf{u}^{n}\right\|_{h}^{2} \leq & \frac{36 \Delta t^{2}}{\Delta x}\left(\left|\alpha_{1}\right|+\left|\alpha_{2}\right|\right)^{2}\left\|\mathbf{u}^{n}\right\|_{h}^{2}\left|\mathbf{u}^{n}\right|_{1, h}^{2}+64 \varepsilon^{4} \frac{\Delta t^{2}}{\Delta x^{4}}\left|\mathbf{u}^{n}\right|_{2, h}^{2} \\
& +144 \frac{\Delta t^{2}}{\Delta x^{4}}\left\|\mathbf{u}^{n}\right\|_{h}^{4}\left|\mathbf{u}^{n}\right|_{1, h}^{2}+\frac{16 \Delta t^{2}}{\Delta x^{2}}\left|\mathbf{u}^{n}\right|_{1, h}^{2}
\end{aligned}
$$

which by (3.52) gives

$$
\begin{aligned}
\left\|\mathbf{u}^{n+1}-\mathbf{u}^{n}\right\|_{h}^{2} \leq & 36 \frac{\Delta t^{2}}{\Delta x}\left(\left|\alpha_{1}\right|+\left|\alpha_{2}\right|\right)^{2}\left\|\mathbf{u}^{n}\right\|_{, h}^{2}\left|\mathbf{u}^{n}\right|_{1, h}^{2}+\Delta t \varepsilon^{2}(1-\delta)\left|\mathbf{u}^{n}\right|_{2, h}^{2} \\
& +144 \frac{\Delta t^{2}}{\Delta x^{4}}\left\|\mathbf{u}^{n}\right\|_{h}^{4}\left|\mathbf{u}^{n}\right|_{1, h}^{2}+\frac{16 \Delta t^{2}}{\Delta x^{2}}\left|\mathbf{u}^{n}\right|_{1, h}^{2} .
\end{aligned}
$$

On substitution of (3.58) back into (3.55), we arrive at

$$
\begin{aligned}
\left\|\mathbf{u}^{n+1}\right\|_{h}^{2}-\exp \left(\frac{\Delta t}{\varepsilon^{2}}\right)\left\|\mathbf{u}^{n}\right\|_{h}^{2}+ & \Delta t \varepsilon^{2} \delta\left|\mathbf{u}^{n}\right|_{2, h}^{2} \leq \frac{16 \Delta t^{2}}{\Delta x^{2}}\left|\mathbf{u}^{n}\right|_{1, h}^{2} \\
& +\frac{36 \Delta t^{2}}{\Delta x}\left(\left(\left|\alpha_{1}\right|+\left|\alpha_{2}\right|\right)^{2}+\frac{4}{\Delta x^{3}}\left\|\mathbf{u}^{n}\right\|_{h}^{2}\right)\left\|\mathbf{u}^{n}\right\|_{h}^{2}\left|\mathbf{u}^{n}\right|_{1, h}^{2} .
\end{aligned}
$$

Using Lemma (2.14) and (3.53), (3.59) can be reduced to

$$
\left\|\mathbf{u}^{n+1}\right\|_{h}^{2}-\exp \left(\frac{k}{\varepsilon^{2}}\right)\left\|\mathbf{u}^{n}\right\|_{h}^{2}+\Delta t \varepsilon^{2} \delta^{2} \eta^{2}\left|\mathbf{u}^{n}\right|_{1, h}^{2} \leq \frac{36 \Delta t^{2}}{\Delta x}\left(\left(\left|\alpha_{1}\right|+\left|\alpha_{2}\right|\right)^{2}+\frac{4}{\Delta x^{3}}\left\|\mathbf{u}^{n}\right\|_{h}^{2}\right)\left\|\mathbf{u}^{n}\right\|_{h}^{2}\left|\mathbf{u}^{n}\right|_{1, h}^{2} .
$$

We then need to show by induction on $n$, that

$$
\left\|\mathbf{u}^{n+1}\right\|_{h}^{2}+\frac{\Delta t}{2} \varepsilon^{2} \delta^{2} \eta^{2}\left|\mathbf{u}^{n}\right|_{1, h}^{2} \leq \exp \left(\frac{\Delta t}{\varepsilon^{2}}\right)\left\|\mathbf{u}^{n}\right\|_{h}^{2} .
$$

For $n=0$, from (3.60), we obtain

$$
\left\|\mathbf{u}^{1}\right\|_{h}^{2}+\Delta t \varepsilon^{2} \delta^{2} \eta^{2}\left|\mathbf{u}^{0}\right|_{1, h}^{2} \leq \exp \left(\frac{\Delta t}{\varepsilon^{2}}\right)\left\|\mathbf{u}^{0}\right\|_{h}^{2}+\frac{36 \Delta t^{2}}{\Delta x}\left[\left(\left|\alpha_{1}\right|+\left|\alpha_{2}\right|\right)^{2}+\frac{4}{\Delta x^{3}}\left\|\mathbf{u}^{0}\right\|_{h}^{2}\right]\left\|\mathbf{u}^{0}\right\|_{h}^{2}\left|\mathbf{u}^{0}\right|_{1, h}^{2},
$$

which with (3.54) leads to

$$
\left\|\mathbf{u}^{1}\right\|_{h}^{2}+\frac{\Delta t}{2} \varepsilon^{2} \delta^{2} \eta^{2}\left|\mathbf{u}^{0}\right|_{1, h}^{2} \leq \exp \left(\frac{\Delta t}{\varepsilon^{2}}\right)\left\|\mathbf{u}^{0}\right\|_{h}^{2},
$$

which is (3.61) for $n=0$. Assuming now that (3.61) is true up the order $n-1$, for $s=0,2, \ldots n-1$, we have

$$
\left\|\mathbf{u}^{s}\right\|_{h}^{2} \leq \exp \left(\frac{\Delta t}{\varepsilon^{2}}\right)\left\|\mathbf{u}^{s-1}\right\|_{h}^{2} \text { and }\left\|\mathbf{u}^{s}\right\|_{h}^{2} \leq \exp \left(\frac{s \Delta t}{\varepsilon^{2}}\right)\left\|\mathbf{u}^{0}\right\|_{h}^{2} .
$$

Using (3.63)in (3.60), one obtains

$$
\begin{aligned}
\left\|\mathbf{u}^{n+1}\right\|_{h}^{2}+\Delta t \varepsilon^{2} \delta^{2} \eta^{2}\left|\mathbf{u}^{n}\right|_{1, h}^{2} & \leq \exp \left(\frac{\Delta t}{\varepsilon^{2}}\right)\left\|\mathbf{u}^{n}\right\|_{h}^{2} \\
& +\frac{36 \Delta t}{\Delta x} \exp \left(\frac{2 n \Delta t}{\varepsilon^{2}}\right)\left[\left(\left|\alpha_{1}\right|+\left|\alpha_{2}\right|\right)^{2}+\frac{4}{\Delta x^{3}}\left\|\mathbf{u}^{0}\right\|_{h}^{2}\right]\left\|\mathbf{u}^{0}\right\|_{h}^{2}\left|\mathbf{u}^{n}\right|_{1, h}^{2},
\end{aligned}
$$

which by (3.54) gives

$$
\left\|\mathbf{u}^{n+1}\right\|_{h}^{2}+\Delta t \varepsilon^{2} \delta^{2} \eta^{2}\left|\mathbf{u}^{n}\right|_{1, h}^{2} \leq \exp \left(\frac{\Delta t}{\varepsilon^{2}}\right)\left\|\mathbf{u}^{n}\right\|_{h}^{2}+\frac{\Delta t}{2} \varepsilon^{2} \delta^{2} \eta^{2}\left|\mathbf{u}^{n}\right|_{1, h}^{2}
$$


re-written as follows

$$
\left\|\mathbf{u}^{n+1}\right\|_{h}^{2}+\frac{\Delta t}{2} \varepsilon^{2} \delta^{2} \eta^{2}\left|\mathbf{u}^{n}\right|_{1, h}^{2} \leq \exp \left(\frac{\Delta t}{\varepsilon^{2}}\right)\left\|\mathbf{u}^{n}\right\|_{h}^{2}
$$

from the induction assumption, one sees

$$
\begin{aligned}
\left\|\mathbf{u}^{n+1}\right\|_{h}^{2} & \leq \exp \left(\frac{\Delta t}{\varepsilon^{2}}\right)\left\|\mathbf{u}^{n}\right\|_{h}^{2}-\frac{\Delta t}{2} \varepsilon^{2} \delta^{2} \eta^{2}\left|\mathbf{u}^{n}\right|_{1, h}^{2} \\
& \leq \exp \left(\frac{\Delta t}{\varepsilon^{2}}\right)\left[\exp \left(\frac{\Delta t}{\varepsilon^{2}}\right)\left\|\mathbf{u}^{n-1}\right\|_{h}^{2}-\frac{\Delta t}{2} \varepsilon^{2} \delta^{2} \eta^{2}\left|\mathbf{u}^{n-1}\right|_{1, h}^{2}\right]-\frac{\Delta t}{2} \varepsilon^{2} \delta^{2} \eta^{2}\left|\mathbf{u}^{n}\right|_{1, h}^{2} \\
& \vdots \\
& \leq \exp \left(\frac{(n+1) \Delta t}{\varepsilon^{2}}\right)\left\|\mathbf{u}^{0}\right\|_{h}^{2}-\frac{\Delta t}{2} \varepsilon^{2} \delta^{2} \eta^{2} \sum_{s=0}^{n} \exp \left(\frac{(n-s) \Delta t}{\varepsilon^{2}}\right)\left|\mathbf{u}^{s}\right|_{1, h}^{2} .
\end{aligned}
$$

Hence we have

$$
\left\|\mathbf{u}^{n+1}\right\|_{h}^{2}+\frac{\Delta t}{2} \varepsilon^{2} \delta^{2} \eta^{2} \sum_{s=0}^{n} \exp \left(\frac{(n-s) \Delta t}{\varepsilon^{2}}\right)\left|\mathbf{u}^{s}\right|_{1, h}^{2} \leq \exp \left(\frac{(n+1) \Delta t}{\varepsilon^{2}}\right)\left\|\mathbf{u}^{0}\right\|_{h}^{2}
$$

Therefore, the proof is complete.

\section{Multilevel finite volume approximation}

This section is an application of the method presented in [4], in which the shallow water equations is analyzed. Here, we are concerned with the convective Cahn-Hilliard equation (1.1)-(1.3). We formulate in the spirit of [4] two methods approximating (1.1)-(1.3), namely: implicit multilevel finite volume method and explicit multilevel finite volume method. These new methods are next studied thoroughly. We first examine the stability analysis of the implicit multilevel method and then the existence of unique solution and the convergence analysis of the method. Lastly, we provide conditions under which the explicit multilevel method is stable.

To make this text self contained for the reader, we recall below the multilevel finite volume approximation as described in Bousquet et. al. [4]. Let $N_{0}$ and $M_{0}$ be integers such that $3 N_{0} \Delta x=2 L$ and $\Delta t M_{0}=T$. We discretize $\mathcal{M}$ into fine meshes and coarser meshes. The fine mesh is consisting of $3 N_{0}$ cells $\left(k_{i}\right)_{1 \leq i \leq 3 N_{0}}$ of uniform length $\Delta x$ and centres $x_{i}$, where

$$
x_{i}=(i-1) \Delta x+\frac{\Delta x}{2}-L, 1 \leq i \leq 3 N_{0} .
$$

The coarse mesh is consisting of $N_{0}$ cells $\left(K_{l}\right)_{1 \leq l \leq N_{0}}$ of uniform length $3 h$, where

$$
K_{l}=\left(x_{3 l-2-1 / 2}, x_{3 l+1 / 2}\right) .
$$

The approximation of the cell average, denoted by $u_{i}$, on the fine grid, $1 \leq i \leq 3 N_{0}$, is given by

$$
\frac{d}{d t} u_{i}(t)-\left(C_{h}(\mathbf{u}(t), \tilde{\mathbf{u}}(t))\right)_{i}+\varepsilon^{2} \Delta_{h}^{2} u_{i}(t)=\Delta_{h} f\left(u_{i}(t)\right)
$$

The approximations $U$ on the coarse mesh is given by

$$
U_{l}=\frac{1}{3}\left[u_{3 l-2}+u_{3 l-1}+u_{3 l}\right], 1 \leq l \leq N_{0},
$$


and the incremental unknowns are defined by

$$
\begin{cases}Z_{3 l-2} & =u_{3 l-2}-U_{l} \\ Z_{3 l-1} & =u_{3 l-1}-U_{l} \\ Z_{3 l} & =u_{3 l}-U_{l}\end{cases}
$$

From (4.2), it is observed that $\sum_{\alpha=0}^{2} Z_{3 l-\alpha}=0$, and by inverting the system, one can obtain

$$
\begin{cases}u_{3 l-2} & =U_{l}+Z_{3 l-2} \\ u_{3 l-1} & =U_{l}-Z_{3 l-2}-Z_{3 l} \\ u_{3 l} & =U_{l}+Z_{3 l}\end{cases}
$$

We use the following discretization for Eq. (1.1), $1 \leq l \leq N_{0}$, on the coarse grid:

$$
\frac{d U_{l}}{d t}(t)-\left(C_{3 h}(U(t), \tilde{U}(t))\right)_{l}+\varepsilon^{2} \Delta_{3 h}^{2} U_{l}(t)=\Delta_{3 h} f\left(U_{l}(t)\right),
$$

where $U_{l}$ is the approximation of the cell averages for $U$ over $K_{l}$.

Let $p>1$ and $q>1$ be two fixed integers. We discretize (4.1) on the finer mesh by using time step $\Delta t / p$ and equation (4.4) on the coarser mesh by using time step $\Delta t$.

We assume that $n$ is a multiple of $q+1$ and $\left(u_{i}^{n}\right)_{1 \leq i \leq 3 N_{0}}$ are known, where $u_{i}^{n}$ is an approximation of the average value of $u$ over $k_{i}$ at the grid $t=n \Delta t$, for $i=1, \ldots, 3 N_{0}$. For $0 \leq r \leq p$ and $1 \leq m \leq q+1$, we let $u_{i}^{n+r / p}$ be the approximate solution of the mean values over $k_{i}$ at time $t_{n+t / p}=n \Delta t+r \Delta t / p$ for $i=1, \ldots, 3 N_{0}$ and $U_{l}^{n+m}$ the approximate solution of the mean value on the coarse mesh $K_{l}$ at time $t_{n+m}=(n+m) \Delta t$ for $l=1, \ldots, N_{0}$.

\subsection{Implicit multilevel schemes}

Here we apply the implicit finite volume method discussed in section 3 to discretize (4.1) on the fine mesh and (4.4) on the coarse mesh. For $r=0,1 \ldots, p-1$ and $s=1,2, \ldots, q$, the following multilevel scheme is used to discretize Eqs. (1.1)-(1.3).

$$
\begin{aligned}
& \frac{p}{\Delta t}\left(u_{i}^{n+(r+1) / p}-u_{i}^{n+r / p}\right)-\left(C_{h}\left(\mathbf{u}^{n+(r+1) / p}, \tilde{\mathbf{u}}^{n+r / p}\right)\right)_{i}+\varepsilon^{2} \Delta_{h}^{2} u_{i}^{n+(r+1) / p}=\nabla_{h}^{+}\left[\varphi_{i-\frac{1}{2}}^{n+r / p} \nabla_{h}^{-} u_{i}^{n+(r+1) / p}\right] \\
& \frac{U_{l}^{n+m+1}-U_{l}^{n+m}}{\Delta t}-\left(C_{3 h}\left(\mathbf{U}^{n+m+1}, \tilde{\mathbf{U}}^{n+m}\right)\right)_{l}+\varepsilon^{2} \Delta_{3 h}^{2} U_{l}^{n+m+1}=\nabla_{3 h}^{+}\left[\Phi_{l-\frac{1}{2}}^{n+m} \nabla_{3 h}^{-} U_{l}^{n+m+1}\right] \\
& u_{i}^{n+(r+1) / p}=u_{i+3 N_{0}}^{n+(r+1) / p} \\
& U_{l}^{n+m+1}=U_{l+N_{0}}^{n+m+1} \\
& u_{i}^{0}=\frac{1}{\Delta x} \int_{k_{i}} u^{0}(x) d x
\end{aligned}
$$

where $1 \leq i \leq 3 N_{0}$ and $1 \leq l \leq N_{0}$ and $\Phi_{l-\frac{1}{2}}^{n+m}=\frac{f^{\prime}\left(\mathbf{U}_{l}^{n+m}\right)+f^{\prime}\left(\mathbf{U}_{l-1}^{n+m}\right)}{2}$.

The multilevel discretization consists in alternating $p$ steps on (4.5a) with smaller time step $\Delta t / p$, from $t_{n}$ to $t_{n+1}$ and then $q$ steps on (4.5b) with time step $\Delta t$, the incremental being frozen at $t_{n+1}$ from $t_{n+1}$ to $t_{n+q+1}$. Then, using Eq. (4.3), we go back to the finer mesh for $p$ steps from $t_{n+q+1}$ to $t_{n+q+2}$.

Theorem 4.1. For $\Delta t<4 \varepsilon^{2}$, then the approximate solution $\boldsymbol{u}^{n}$ of (4.5a) - (4.5e) is unique. 
Proof. Suppose $\mathbf{v}_{0}=\mathbf{u}_{0}$ and let $\mathbf{v}^{1}, \mathbf{v}^{2}, \ldots, \mathbf{v}_{0}^{M} \in \mathcal{H}_{h}, \Delta t M_{0}=T$, such that $\mathbf{v}^{n}$ satisfies (4.5a) and (4.5b) with the relation (4.3). Let $\mathbf{z}^{i}=\mathbf{u}^{i}-\mathbf{v}^{i}$ with $\mathbf{z}^{0}=0$, we then have

$$
\begin{aligned}
\frac{1}{\Delta t}\left(z_{i}^{n+1}-z_{i}^{n}\right)-\left[\left(C_{h}\left(\mathbf{u}^{n+1}, \tilde{\mathbf{u}}^{n}\right)\right)_{i}\right. & \left.-\left(C_{h}\left(\mathbf{v}^{n+1}, \tilde{\mathbf{v}}^{n}\right)\right)_{i}\right]+\varepsilon^{2} \Delta_{h}^{2} z_{i}^{n+1} \\
& =\nabla_{h}^{+}\left[\varphi_{i-\frac{1}{2}}^{n} \nabla_{h}^{-} u_{i}^{n+1}-\psi_{i-\frac{1}{2}}^{n} \nabla_{h}^{-} v_{i}^{n+1}\right] .
\end{aligned}
$$

We use mathematical induction on $n$ and $n$ can be written as $n=s(q+1)+m$ for $0 \leq m \leq q$. Since $\mathbf{z}^{0}=0$, the theorem holds for $n=0$. We assume that $\mathbf{z}^{t}=0$ for $t \leq n$. If $m=0$, we need to show the theorem holds by induction on $r$ using Eq. (4.5a). By assuming that $\mathbf{z}^{n+r / p}=0$, we have

$$
\frac{z_{i}^{n+(r+1) / p}}{\Delta t}-\left(C_{h}\left(\mathbf{z}^{n+(r+1) / p}, \tilde{\mathbf{u}}^{n+r / p}\right)\right)_{i}+\varepsilon^{2} \Delta_{h}^{2} z_{i}^{n+(r+1) / p}=\nabla_{h}^{+}\left(\varphi_{i-\frac{1}{2}}^{n+r / p} \nabla_{h}^{-} z_{i}^{n+1}\right) .
$$

Multiplying (4.6) by $\frac{\Delta t \Delta x}{p} z_{i}^{n+(r+1) / p}$ and summing from $i=1$ to $i=3 N_{0}$, we obtain

$$
\left\|\mathbf{z}^{n+(r+1) / p}\right\|_{h}^{2}+\frac{\Delta t \varepsilon^{2}}{p}\left|\mathbf{z}^{n+(r+1) / p}\right|_{2, h}^{2}=\frac{\Delta t \Delta x}{p} \sum_{i=1}^{N} \nabla_{h}^{+}\left(\varphi_{i-\frac{1}{2}}^{n+r / p} \nabla_{h}^{-} z_{i}^{n+1}\right) .
$$

Hence using Lemmas 2.3 and 2.2, we get

$$
\left(1-\frac{\Delta t}{4 p \varepsilon^{2}}\right)\left\|\mathbf{z}^{n+(r+1) / p}\right\|_{h}^{2} \leq 0,
$$

which for $\frac{\Delta t}{4 p \varepsilon^{2}}<1$, and after repeating $p$ times gives

$$
\left\|\mathbf{z}^{n+1}\right\|_{h}^{2} \leq 0
$$

and implies

$$
\left\|\mathbf{z}^{n+1}\right\|_{h}=0
$$

If $m_{0} \geq 1$, we use Eq. (4.5b) to prove the theorem. We first calculate $\mathbf{U}^{n}$ and $\mathbf{V}^{n}$ using Eq. (4.2) from $\mathbf{u}^{n}$ and $\mathbf{v}^{n}$, respectively, we have

$$
\begin{aligned}
\frac{1}{\Delta t}\left(\mathcal{Z}_{l}^{n+1}-\mathcal{Z}_{l}^{n}\right)-[ & {\left[\left(C_{3 h}\left(\mathbf{U}^{n+1}, \tilde{\mathbf{U}}^{n}\right)\right)_{l}-\left(C_{3 h}\left(\mathbf{V}^{n+1}, \tilde{\mathbf{V}}^{n}\right)\right)_{l}\right]+\varepsilon^{2} \Delta_{3 h}^{2} \mathcal{Z}_{l}^{n+1} } \\
& =\nabla_{3 h}^{+}\left(\Phi_{i-\frac{1}{2}}^{n} \nabla_{3 h}^{-} U_{l}^{n+1}\right)-\nabla_{3 h}^{+}\left(\Psi_{i-\frac{1}{2}}^{n} \nabla_{3 h}^{-} V_{l}^{n+1}\right),
\end{aligned}
$$

where $\mathcal{Z}^{n}=\mathbf{U}^{n}-\mathbf{V}^{n}, \Psi_{i-\frac{1}{2}}^{n}=\frac{f^{\prime}\left(v_{i}^{n}\right)+f^{\prime}\left(v_{i-1}^{n}\right)}{2}$ for $l=1, \ldots, N_{0}$. Using the assumption $\tilde{\mathbf{U}}^{n}=\tilde{\mathbf{V}}^{n}$, (4.7) becomes

$$
\left.\frac{\mathcal{Z}_{l}^{n+1}}{\Delta t}-\left(C_{3 h}\left(\mathcal{Z}^{n+1}\right), \tilde{\mathbf{U}}^{n}\right)\right)_{l}+\varepsilon^{2} \Delta_{3 h}^{2} \mathcal{Z}_{l}^{n+1}=\nabla_{3 h}^{+}\left(\Phi_{i-\frac{1}{2}}^{n} \nabla_{3 h}^{-} \mathcal{Z}_{l}^{n+1}\right)
$$

Multiplying Eq. (4.7) by $3 \Delta t \Delta x \mathcal{Z}_{l}^{n+1}$ and adding the corresponding equalities for $l=1, \cdots, N_{0}$, we obtain

$$
\left\|\mathcal{Z}^{n+1}\right\|_{3 h}^{2}+\Delta t \varepsilon^{2}\left|\mathcal{Z}^{n+1}\right|_{2,3 h}^{2}=3 \Delta t \Delta x \sum_{l=1}^{N_{0}} \nabla_{3 h}^{+}\left(\Phi_{i-\frac{1}{2}}^{n} \nabla_{3 h}^{-} \mathcal{Z}_{l}^{n+1}\right) \mathcal{Z}_{l}^{n+1}
$$

Hence using Lemma 2.3, one obtains

$$
\left\|\mathcal{Z}^{n+1}\right\|_{3 h}^{2}+\Delta t \varepsilon^{2}\left|\mathcal{Z}^{n+1}\right|_{2,3 h}^{2} \leq \Delta t\left|\mathcal{Z}^{n+1}\right|_{1,3 h}^{2}
$$


Applying Lemma 2.2 and Young's inequality, (4.8) leads to

$$
\left(1-\frac{\Delta t}{4 \varepsilon^{2}}\right)\left\|\mathcal{Z}^{n+1}\right\|_{3 h}^{2} \leq 0
$$

which for $\frac{\Delta t}{4 \varepsilon^{2}}<1$, gives $\mathcal{Z}^{n+1}=0$. Therefore, $\mathbf{z}^{n+1}=0$. This completes the proof of Theorem 4.1.

Theorem 4.2. The multilevel method defined by the equations (4.5a) -(4.5e) is conditionally stable in $L^{\infty}\left(0, T ; \mathcal{H}_{h}\right)$ in the sense that if $\Delta t \leq \varepsilon^{2}$, then

$$
\left\|\boldsymbol{u}^{n}\right\|_{h}^{2} \leq 2^{\frac{2 T}{\varepsilon^{2}}}\left\|\boldsymbol{u}^{0}\right\|_{h}^{2}
$$

Proof. By multiplying (4.5a) by $2 \frac{\Delta t \Delta x}{p} u_{i}^{n+(r+1) / p}$ and adding the corresponding equalities for $i=1, \ldots, 3 N_{0}$, we obtain

$$
\begin{aligned}
2 \Delta x \sum_{i=1}^{3 N_{0}}\left(u_{i}^{n+(r+1) / p}-u_{i}^{n+r / p}\right) u_{i}^{n+(r+1) / p} & +2 \Delta x \frac{\Delta t}{p} \varepsilon^{2} \sum_{i=1}^{3 N_{0}} \Delta_{h}^{2} u_{i}^{n+(r+1) / p} u_{i}^{n+(r+1) / p} \\
& =2 \Delta x \frac{\Delta t}{p} \sum_{i=1}^{3 N_{0}} \nabla_{h}^{+}\left[\varphi_{i-\frac{1}{2}}^{n+r / p} \nabla_{h}^{-} u_{i}^{n+(r+1) / p}\right] u_{i}^{n+(r+1) / p} .
\end{aligned}
$$

Using Lemma 2.3, Eq. (4.9) becomes

$$
\left\|\mathbf{u}^{n+(r+1) / p}\right\|_{h}^{2}-\left\|\mathbf{u}^{n+r / p}\right\|_{h}^{2}+\left\|\mathbf{u}^{n+(r+1) / p}-\mathbf{u}^{n+r / p}\right\|_{h}^{2}+2 \frac{\Delta t}{p} \varepsilon^{2}\left|\mathbf{u}^{n+(r+1) / p}\right|_{2, h}^{2} \leq \frac{2 \Delta t}{p}\left|\mathbf{u}^{n+(r+1) / p}\right|_{1, h}^{2}
$$

Applying Lemma 2.2 together with Young's inequality, (4.10) becomes

$$
\left\|\mathbf{u}^{n+(r+1) / p}\right\|_{h}^{2}-\left\|\mathbf{u}^{n+r / p}\right\|_{h}^{2}+\left\|\mathbf{u}^{n+(r+1) / p}-\mathbf{u}^{n+r / p}\right\|_{h}^{2} \leq \frac{\Delta t}{2 p \varepsilon^{2}}\left\|\mathbf{u}^{n+(r+1) / p}\right\|_{h}^{2} .
$$

Thus we have

$$
\left[1-\frac{\Delta t}{2 p \varepsilon^{2}}\right]\left\|\mathbf{u}^{n+(r+1) / p}\right\|_{h}^{2} \leq\left\|\mathbf{u}^{n+r / p}\right\|_{h}^{2}
$$

Based on (2.7), for

$$
\frac{\Delta t}{2 p \varepsilon^{2}} \leq \frac{1}{2}
$$

we have

$$
\left\|\mathbf{u}^{n+(r+1) / p}\right\|_{h}^{2} \leq 2^{\frac{2 \Delta t}{p \varepsilon^{2}}}\left\|\mathbf{u}^{n+r / p}\right\|_{h}^{2}
$$

After $p$ iterations, we obtain

$$
\left\|\mathbf{u}^{n+1}\right\|_{h}^{2} \leq 2^{\frac{2 \Delta t}{\varepsilon^{2}}}\left\|\mathbf{u}^{n}\right\|_{h}^{2} .
$$

We now perform $q$ iterations on the coarse grid, Eq. (4.5b), using time step $\Delta t$ and the relations (4.3). At time $t_{n+m}=(n+m) \Delta t, 2 \leq m \leq q+1$, the incremental unknowns $Z_{i}$ are frozen at time $(n+1) \Delta t$. 
Multiplying Eq. (4.5b) by $6 \Delta x \Delta t U_{l}^{n+m+1}$ and adding the equalities for $l=1, \ldots N_{0}$, we get

$$
\begin{aligned}
6 \Delta x \sum_{l=1}^{N}\left(U_{l}^{n+m+1}-U_{l}^{n+m}\right) U_{l}^{n+m+1} & +6 \Delta x \Delta t \varepsilon^{2} \sum_{l=1}^{N} \Delta_{3 h}^{2} \mathbf{U}^{n+m+1} U_{l}^{n+m+1} \\
& =6 \Delta x \Delta t \sum_{l=1}^{N} \nabla_{3 h}^{+}\left[\Phi_{l-\frac{1}{2}}^{n+m} \nabla_{3 h}^{-} \mathbf{U}^{n+m+1}\right] U_{l}^{n+m+1} .
\end{aligned}
$$

Using Lemma 2.3 together with (4.13), we get

$$
\left\|\mathbf{U}^{n+m+1}\right\|_{3 h}^{2}-\left\|\mathbf{U}^{n+m}\right\|_{3 h}^{2}+\left\|\mathbf{U}^{n+m+1}-\mathbf{U}^{n+m}\right\|_{3 h}^{2}+2 \Delta t \varepsilon^{2}\left|\mathbf{U}^{n+m+1}\right|_{2,3 h}^{2} \leq 2 \Delta t\left|\mathbf{U}^{n+m+1}\right|_{1,3 h}^{2} .
$$

Using Lemma 2.2 and Young's inequality, (4.14) becomes

$$
\left\|\mathbf{U}^{n+m+1}\right\|_{3 h}^{2}-\left\|\mathbf{U}^{n+m}\right\|_{3 h}^{2}+\left\|\mathbf{U}^{n+m+1}-\mathbf{U}^{n+m}\right\|_{3 h}^{2} \leq \frac{\Delta t}{2 \varepsilon^{2}}\left\|\mathbf{U}^{n+m+1}\right\|_{3 h}^{2} .
$$

Thus we have

$$
\left[1-\frac{\Delta t}{2 \varepsilon^{2}}\right]\left\|\mathbf{U}^{n+m+1}\right\|_{3 h}^{2} \leq\left\|\mathbf{U}^{n+m}\right\|_{3 h}^{2}
$$

Using (2.7)), for

$$
\frac{\Delta t}{2 \varepsilon^{2}} \leq \frac{1}{2}
$$

we obtain

$$
\left\|\mathbf{U}^{n+m+1}\right\|_{3 h}^{2} \leq 2^{\frac{2 \Delta t}{\varepsilon^{2}}}\left\|\mathbf{U}^{n+m}\right\|_{3 h}^{2} .
$$

since the incremental unknowns are frozen ate $t_{n+1}$, we have $\mathbf{Z}^{n+s+1}=\mathbf{Z}^{n+1}$, for $s=1, \cdots, q$. From the definition of the increments $Z_{3 l-\alpha}^{n+1}$, Eq. (4.2), we have

$$
u_{3 l-\alpha}^{n+m}=U_{l}^{n+m}+Z_{3 l-\alpha}^{n+1}, \quad 1 \leq l \leq N_{0}, \quad \alpha=0,1,2 .
$$

Taking the sum over $\alpha$, we get

$$
\sum_{\alpha=0}^{2}\left|u_{3 l+\alpha}^{n+m}\right|^{2}=\sum_{\alpha=0}^{2}\left|U_{l}^{n+m}+Z_{3 l-\alpha}^{n+1}\right|^{2}=3\left|U_{l}^{n+m}\right|^{2}+\sum_{\alpha=0}^{2}\left|Z_{3 l-\alpha}^{n+1}\right|^{2} .
$$

For $m=1, \ldots q+1$, the following relation holds

$$
\left\|\mathbf{u}^{n+m}\right\|_{h}^{2}=\left\|\mathbf{U}^{n+m}\right\|_{3 h}^{2}+\left\|\mathbf{Z}^{n+1}\right\|_{h}^{2}
$$

By adding $\left\|\mathbf{Z}^{n+1}\right\|_{h}^{2}$ to both sides of inequality (4.16) and using (4.17), we get

$$
\left\|\mathbf{u}^{n+m+1}\right\|_{h}^{2} \leq 2^{\frac{2 \Delta t}{\varepsilon^{2}}}\left\|\mathbf{u}^{n+m}\right\|_{h}^{2} .
$$

After $q$ iterations, we have

$$
\left\|\mathbf{u}^{n+q+1}\right\|_{h}^{2} \leq 2^{\frac{2 \Delta t q}{\varepsilon^{2}}}\left\|\mathbf{u}^{n+1}\right\|_{h}^{2}
$$

and using (4.12)

$$
\left\|\mathbf{u}^{n+q+1}\right\|_{h}^{2} \leq 2^{\frac{2 \Delta t(q+1)}{\varepsilon^{2}}}\left\|\mathbf{u}^{n}\right\|_{h}^{2} .
$$

By induction over $n$, we have

$$
\left\|\mathbf{u}^{n}\right\|_{h}^{2} \leq 2^{\frac{2 n \Delta t}{\varepsilon^{2}}}\left\|\mathbf{u}^{0}\right\|_{h}^{2} \leq 2^{\frac{2 T}{\varepsilon^{2}}}\left\|\mathbf{u}^{0}\right\|_{h}^{2}
$$

This completes the proof. 
Remark 4.1. It should be noted that the stability condition $\Delta t \leq \varepsilon^{2}$ includes the condition of existence/uniqueness of solution $\Delta t \leq 4 \varepsilon^{2}$.

About the convergence of the scheme presented, we claim that

Theorem 4.3. Suppose that the solution $u(x, t)$ of Eq. (1.1) is sufficiently smooth. Assume that $\Delta t, \Delta x$ are given such that the conditions below are satisfied. Then, the solution of the finite volume discretization (4.5a) - (4.5e) converges to the solution of Eq. (1.1) in the discrete $L^{2}$-norm with rate of convergence $\mathcal{O}\left(\Delta t+(3 \Delta x)^{2}\right)$.

The proof of this result uses the same techniques presented in the proof of Theorem 4.2, and Theorem 3.3. There are positive constants $c_{1}, c_{2}, c_{3}$ such that

$$
\begin{aligned}
& \Delta t<4 \varepsilon^{2}, \Delta t c_{1} \leq \min \left(\frac{1}{2}, \frac{p}{2}\right) \\
& \frac{3 c_{2}}{\Delta x}\left(\frac{\Delta t}{p}+\Delta x^{2}\right)^{2} \leq \frac{1}{2 T} \varepsilon^{2} \eta^{2} 4^{-T} \exp \left(-T c_{3}\right) \\
& \frac{c_{2}}{\Delta x}\left(\frac{\Delta t}{p}+(3 \Delta x)^{2}\right)^{2} \leq \frac{1}{2 T} \varepsilon^{2} \eta^{2} 4^{-T} \exp \left(-T c_{3}\right) .
\end{aligned}
$$

Remark 4.2. The stability conditions for the implicit one-level and multilevel methods are given below:

- The one-level finite volume method on the fine mesh is stable when $\frac{\Delta t}{p} \leq \varepsilon^{2}$.

- The one-level finite volume method on the coarse mesh is stable when $\Delta t \leq \varepsilon^{2}$.

- The multilevel finite volume method is stable when both (4.11) and (4.15) are satisfied, i.e., $\Delta t \leq \varepsilon^{2}$.

Hence we see that the stability condition of both the one-level on the coarse mesh and multilevel method is the same.

Remark 4.3. Remark 3.2 applies for Theorem 4.3.

\subsection{Explicit multilevel finite volume method}

For $0 \leq r \leq p-1$ and $1 \leq m \leq q$, we discretize Eq. (1.1) using explicit multilevel finite volume method.

$$
\begin{aligned}
& \frac{p}{\Delta t}\left(u_{i}^{n+(r+1) / p}-u_{i}^{n+r / p}\right)-\left(C_{h}\left(\mathbf{u}^{n+r / p}, \mathbf{u}^{n+r / p}\right)\right)_{i}+\varepsilon^{2} \Delta_{h}^{2} u_{i}^{n+r / p}=\nabla_{h}^{+}\left[\varphi_{i-\frac{1}{2}}^{n+r / p} \nabla_{h}^{-} u_{i}^{n+r / p}\right], \\
& \frac{U_{l}^{n+m+1}-U_{l}^{n+m}}{\Delta t}-\left(C_{3 h}\left(\mathbf{U}^{n+m}, \mathbf{U}^{n+m}\right)\right)_{l}+\varepsilon^{2} \Delta_{3 h}^{2} U_{l}^{n+m}=\nabla_{3 h}^{+}\left[\Phi_{i-\frac{1}{2}}^{n+r / p} \nabla_{3 h}^{-} U_{l}^{n+m}\right] . \\
& u_{i}^{n+r / p}=u_{i+3 N_{0}}^{n+r / p}, \\
& U_{l}^{n+s}=U_{l+N_{0}}^{n+s}, \\
& u_{i}^{0}=\frac{1}{\Delta x} \int_{k_{i}} u^{0}(x) d x,
\end{aligned}
$$

where $1 \leq i \leq 3 N_{0}, 1 \leq l \leq N_{0}$. Since it is explicit, we have a unique solution. But as far as the stability is concerned, we claim that 
Theorem 4.4. We assume that the following satisfied for some $\delta, 0<\delta<1$ :

$$
\begin{aligned}
& \frac{32 \Delta t}{\Delta x^{4}} \leq \frac{1-\delta}{2 \varepsilon^{2}} \min \{p, 81\} \\
& \frac{16 \Delta t}{\Delta x^{2}} \leq \varepsilon^{2} \delta \eta^{2}(1-\delta) \min \{p, 9\} \\
& \frac{72 \Delta t}{p \Delta x}\left(\left(\left|\alpha_{1}\right|+\left|\alpha_{2}\right|\right)^{2}+\frac{4}{\Delta x^{3}}\left\|\boldsymbol{u}^{0}\right\|_{h}^{2}\right)\left\|\boldsymbol{u}^{0}\right\|_{h}^{2} \leq \varepsilon^{2} \delta^{2} \eta^{2} \exp \left(\frac{-2 T}{\varepsilon^{2}}\right), \\
& \frac{24 \Delta t}{\Delta x}\left(\left(\left|\alpha_{1}\right|+\left|\alpha_{2}\right|\right)^{2}+\frac{4}{27 \Delta x^{3}}\left\|\boldsymbol{u}^{0}\right\|_{h}^{2}\right)\left\|\boldsymbol{u}^{0}\right\|_{h}^{2} \leq \varepsilon^{2} \delta^{2} \eta^{2} \exp \left(\frac{-2 T}{\varepsilon^{2}}\right) .
\end{aligned}
$$

Then the multilevel method defined by the equations $(4.18 \mathrm{a})-(4.18 \mathrm{e})$ is stable in $L^{\infty}\left(0, T ; \mathcal{H}_{h}\right)$ in the following sense:

$$
\begin{aligned}
& \left\|\boldsymbol{u}^{n}\right\|^{2} \leq \exp \left(\frac{\Delta t}{\varepsilon^{2}}\right)\left\|\boldsymbol{u}^{n-1}\right\|^{2} \leq \cdots \leq \exp \left(\frac{n \Delta t}{\varepsilon^{2}}\right)\left\|\boldsymbol{u}^{0}\right\|^{2} \leq \exp \left(\frac{T}{\varepsilon^{2}}\right)\left\|\boldsymbol{u}^{0}\right\|^{2}, \quad n=1,2, \cdots, M_{0}, \\
& \left\|\boldsymbol{u}^{s(q+1)+r / p}\right\|^{2} \leq \exp \left(\frac{r \Delta t}{p \varepsilon^{2}}\right)\left\|\boldsymbol{u}^{s(q+1)}\right\|^{2}, \quad r=1,2, \cdots, p
\end{aligned}
$$

Proof. To prove this assertion we use the approach discussed in section 3 for the one-level explicit method. We assume $n$ is a multiple of $q+1$. Multiplying Eq. (4.18a) by $2 \frac{\Delta t}{p} h u_{i}^{n+r / p}$ and taking the sum from $i=1$ to $3 N_{0}$ together with (2.6) and Lemma 2.2, we obtain

$$
\left\|\mathbf{u}^{n+(r+1) / p}\right\|_{h}^{2}-\left\|\mathbf{u}^{n+(r+1) / p}-\mathbf{u}^{n+r / p}\right\|_{h}^{2}+\frac{\Delta t \varepsilon^{2}}{p}\left|\mathbf{u}^{n+r / p}\right|_{2, h}^{2} \leq \exp \left(\frac{\Delta t}{p \varepsilon^{2}}\right)\left\|\mathbf{u}^{n+r / p}\right\|_{h}^{2}
$$

To estimate the term $\left\|\mathbf{u}^{n+(r+1) / p}-\mathbf{u}^{n+r / p}\right\|_{h}^{2}$, we multiply Eq. (4.18a) by $2 \frac{\Delta t \Delta x}{p}\left(u_{i}^{n+(r+1) / p}-u_{i}^{n+r / p}\right)$ and summing from $i=1$ to $i=3 N_{0}$, we find

$$
\begin{gathered}
\left\|\mathbf{u}^{n+(r+1) / p}-\mathbf{u}^{n+r / p}\right\|_{h}^{2} \leq \\
\frac{36 \Delta t^{2}}{p^{2} \Delta x}\left(\left|\alpha_{1}\right|+\left|\alpha_{2}\right|\right)^{2}\left\|\mathbf{u}^{n+r / p}\right\|_{h}^{2}\left|\mathbf{u}^{n+r / p}\right|_{1, h}^{2}+\frac{64 \Delta t^{2}}{p^{2} h^{4}} \varepsilon^{4}\left|\mathbf{u}^{n+r / p}\right|_{2, h}^{2} \\
\quad+\frac{144 \Delta t^{2}}{p^{2} \Delta x^{4}}\left\|\mathbf{u}^{n+r / p}\right\|_{h}^{4}\left|\mathbf{u}^{n}\right|_{1, h}^{2}+\frac{16 \Delta t^{2}}{p^{2} \Delta x^{2}}\left|\mathbf{u}^{n+r / p}\right|_{1, h}^{2} .
\end{gathered}
$$

Using (4.19), we obtain

$$
\begin{aligned}
\left\|\mathbf{u}^{n+(r+1) / p}-\mathbf{u}^{n+r / p}\right\|_{h}^{2} \leq & \frac{36 \Delta t^{2}}{p^{2} \Delta x}\left(\left|\alpha_{1}\right|+\left|\alpha_{2}\right|\right)^{2}\left\|\mathbf{u}^{n}\right\|_{h}^{2}\left|\mathbf{u}^{n+r / p}\right|_{1, h}^{2}+\frac{\Delta t}{p} \varepsilon^{2}(1-\delta)\left|\mathbf{u}^{n+r / p}\right|_{2, h}^{2} \\
& +144 \frac{\Delta t^{2}}{p^{2} \Delta x^{4}}\left\|\mathbf{u}^{n+r / p}\right\|_{h}^{4}\left|\mathbf{u}^{n+r / p}\right|_{1, h}^{2}+\frac{16 \Delta t^{2}}{p^{2} \Delta x^{2}}\left|\mathbf{u}^{n+r / p}\right|_{1, h}^{2} .
\end{aligned}
$$

On substitution of (4.26) into (4.25), we get

$$
\begin{aligned}
\left\|\mathbf{u}^{n+(r+1) / p}\right\|_{h}^{2}+ & \frac{\Delta t}{p} \varepsilon^{2} \delta\left|\mathbf{u}^{n+r / p}\right|_{2, h}^{2} \leq \exp \left(\frac{\Delta t}{p \varepsilon^{2}}\right)\left\|\mathbf{u}^{n+r / p}\right\|_{h}^{2} \\
& +\frac{36 \Delta t^{2}}{p^{2} \Delta x}\left[\left(\left|\alpha_{1}\right|+\left|\alpha_{2}\right|\right)^{2}+\frac{144}{\Delta x^{3}}\left\|\mathbf{u}^{n+r / p}\right\|_{h}^{2}\right]\left\|\mathbf{u}^{n+r / p}\right\|_{h}^{2}\left|\mathbf{u}^{n+r / p}\right|_{1, h}^{2}
\end{aligned}
$$

Using Lemma (2.14) and (4.20), we obtain

$$
\begin{aligned}
\left\|\mathbf{u}^{n+(r+1) / p}\right\|_{h}^{2} & -\exp \left(\frac{\Delta t}{p \varepsilon^{2}}\right)\left\|\mathbf{u}^{n+r / p}\right\|_{h}^{2}+\frac{\Delta t}{p} \varepsilon^{2} \delta^{2} \eta^{2}\left|\mathbf{u}^{n+r / p}\right|_{1, h}^{2} \\
& -\frac{36 \Delta t^{2}}{p^{2} \Delta x}\left[\left(\left|\alpha_{1}\right|+\left|\alpha_{2}\right|\right)^{2}+\frac{144}{\Delta x^{3}}\left\|\mathbf{u}^{n+r / p}\right\|_{h}^{2}\right]\left\|\mathbf{u}^{n+r / p}\right\|_{h}^{2}\left|\mathbf{u}^{n+r / p}\right|_{1, h}^{2} \leq 0 .
\end{aligned}
$$


In a similar fashion, from (4.18b) together with the assumptions (4.19), (4.20) and (4.22), we obtain

$$
\begin{aligned}
\left\|\mathbf{U}^{n+m+1}\right\|_{3 h}^{2} & -\exp \left(\frac{\Delta t}{\varepsilon^{2}}\right)\left\|\mathbf{U}^{n+m}\right\|_{3 h}^{2}+\Delta t \varepsilon^{2} \delta^{2} \eta^{2}\left|\mathbf{U}^{n+m}\right|_{1,3 h}^{2} \\
& \leq \frac{36 \Delta t^{2}}{3 \Delta x}\left[\left(\left|\alpha_{1}\right|+\left|\alpha_{2}\right|\right)^{2}+\frac{4}{27 \Delta x^{3}}\left\|\mathbf{U}^{n+m}\right\|_{3 h}^{2}\right]\left\|\mathbf{U}^{n+m}\right\|_{3 h}^{2}\left|\mathbf{U}^{n+m}\right|_{1,3 h}^{2} .
\end{aligned}
$$

Now we need to prove the following by induction on $n$

$$
\begin{gathered}
\left\|\mathbf{u}^{n+(r+1) / p}\right\|_{h}^{2}+\frac{\Delta t}{2 p} \varepsilon^{2} \delta^{2} \eta^{2}\left|\mathbf{u}^{n+r / p}\right|_{1, h}^{2} \leq \exp \left(\frac{\Delta t}{p \varepsilon^{2}}\right)\left\|\mathbf{u}^{n+r / p}\right\|_{h}^{2}, \quad \text { for } r=0,1, \ldots, p-1 \\
\left\|\mathbf{U}^{n+m+1}\right\|_{3 h}^{2}+\frac{\Delta t}{2} \varepsilon^{2} \delta^{2} \eta^{2}\left|\mathbf{U}^{n+m}\right|_{1,3 h}^{2} \leq \exp \left(\frac{\Delta t}{\varepsilon^{2}}\right)\left\|\mathbf{U}^{n+m}\right\|_{3 h}^{2}, \quad \text { for } m=1,2, \ldots, q
\end{gathered}
$$

We first show (4.29) and (4.30) hold by induction on $r$ and $m$ when $n=0$. We first show

$$
\left\|\mathbf{u}^{1}\right\|_{h}^{2}+\frac{\Delta t}{2 p} \varepsilon^{2} \delta^{2} \eta^{2} \sum_{r=0}^{p-1} \exp \left(\frac{(p-1-r) \Delta t}{p \varepsilon^{2}}\right)\left|\mathbf{u}^{r / p}\right|_{1, h}^{2} \leq \exp \left(\frac{\Delta t}{p \varepsilon^{2}}\right)\left\|\mathbf{u}^{0}\right\|_{h}^{2}
$$

holds.

For $n=0$, the relation (4.27) becomes

$$
\begin{aligned}
\left\|\mathbf{u}^{(r+1) / p}\right\|_{h}^{2}+\frac{\Delta t}{p} \varepsilon^{2} \delta^{2} \eta^{2}\left|\mathbf{u}^{r / p}\right|_{1, h}^{2} & \leq \exp \left(\frac{\Delta t}{p \varepsilon^{2}}\right)\left\|\mathbf{u}^{r / p}\right\|_{h}^{2} \\
& +36 \frac{\Delta t^{2}}{p^{2} \Delta x}\left[\left(\left|\alpha_{1}\right|+\left|\alpha_{2}\right|\right)^{2}+\frac{4}{\Delta x^{3}}\left\|\mathbf{u}^{r / p}\right\|_{h}^{2}\right]\left\|\mathbf{u}^{r / p}\right\|_{h}^{2}\left|\mathbf{u}^{r / p}\right|_{1, h}^{2} .
\end{aligned}
$$

For $r=0$ using (4.21), we get

$$
\left\|\mathbf{u}^{1 / p}\right\|_{h}^{2}+\frac{\Delta t}{2} \varepsilon^{2} \delta^{2} \eta^{2}\left|\mathbf{u}^{0}\right|_{1, h}^{2} \leq \exp \left(\frac{\Delta t}{p \varepsilon^{2}}\right)\left\|\mathbf{u}^{0}\right\|_{h}^{2}
$$

Let us assume that (4.31) holds up to $r-1$. From the assumption for $s=1,2, \ldots, r-1$, we have

$$
\left\|\mathbf{u}^{s / p}\right\|_{h}^{2} \leq\left\|\mathbf{u}^{(s-1) / p}\right\|_{h}^{2}
$$

and

$$
\left\|\mathbf{u}^{s / p}\right\|_{h}^{2} \leq \exp \left(\frac{s \Delta t}{p \varepsilon^{2}}\right)\left\|\mathbf{u}^{0}\right\|_{h}^{2}
$$

The relation (4.27) becomes

$$
\begin{aligned}
\left\|\mathbf{u}^{(r+1) / p}\right\|_{h}^{2}+\frac{\Delta t}{p} \varepsilon^{2} \delta^{2} \eta^{2}\left|\mathbf{u}^{r / p}\right|_{1, h}^{2} & \leq \exp \left(\frac{\Delta t}{p \varepsilon^{2}}\right)\left\|\mathbf{u}^{r / p}\right\|_{h}^{2} \\
& +36 \frac{\Delta t^{2}}{p^{2} \Delta x} \exp \left(\frac{2 r \Delta t}{p \varepsilon^{2}}\right)\left[\left(\left|\alpha_{1}\right|+\left|\alpha_{2}\right|\right)^{2}+\frac{4}{\Delta x^{3}}\left\|\mathbf{u}^{0}\right\|_{h}^{2}\right]\left\|\mathbf{u}^{0}\right\|_{h}^{2}\left|\mathbf{u}^{r / p}\right|_{1, h}^{2} \\
& \leq \exp \left(\frac{\Delta t}{p \varepsilon^{2}}\right)\left\|\mathbf{u}^{r / p}\right\|_{h}^{2}+\frac{\Delta t}{2 p} \varepsilon^{2} \delta^{2} \eta^{2}\left|\mathbf{u}^{r / p}\right|_{1, h}^{2}
\end{aligned}
$$

which shows us that (4.29) is true for $n=0$. From (4.34), we have

$$
\left\|\mathbf{u}^{1}\right\|_{h}^{2}+\frac{\Delta t}{p} \varepsilon^{2} \delta^{2} \eta^{2} \sum_{r=0}^{p-1} \exp \left(\frac{(p-1-r) \Delta t}{p \varepsilon^{2}}\right)\left|\mathbf{u}^{r / p}\right|_{1, h}^{2} \leq \exp \left(\frac{\Delta t}{\varepsilon^{2}}\right)\left\|\mathbf{u}^{0}\right\|_{h}^{2}
$$


which implies

$$
\left\|\mathbf{u}^{1}\right\|_{h}^{2} \leq \exp \left(\frac{\Delta t}{\varepsilon^{2}}\right)\left\|\mathbf{u}^{0}\right\|_{h}^{2}
$$

We then show (4.30) by using induction on $m$ for $n=0$. From the definition of $\mathbf{U}$, we have

$$
\left\|\mathbf{U}^{n}\right\|_{3 h}^{2} \leq\left\|\mathbf{u}^{n}\right\|_{h}^{2} .
$$

For $m=1$, from (4.19), we have

$$
\begin{aligned}
\left\|\mathbf{U}^{2}\right\|_{3 h}^{2} & -\exp \left(\frac{\Delta t}{\varepsilon^{2}}\right)\left\|\mathbf{U}^{1}\right\|_{3 h}^{2}+\Delta t \varepsilon^{2} \delta^{2} \eta^{2}\left|\mathbf{U}^{1}\right|_{1,3 h}^{2} \\
& -\frac{36 \Delta t^{2}}{3 \Delta x}\left[\left(\left|\alpha_{1}\right|+\left|\alpha_{2}\right|\right)^{2}+\frac{4 \Delta t}{27 \Delta x^{3}}\left\|\mathbf{U}^{1}\right\|_{3 h}^{2}\right]\left\|\mathbf{U}^{1}\right\|_{3 h}^{2}\left|\mathbf{U}^{1}\right|_{1,3 h}^{2} \leq 0 .
\end{aligned}
$$

Then using (4.35) and (4.36), we have

$$
\begin{aligned}
\left\|\mathbf{U}^{2}\right\|_{3 h}^{2} & -\exp \left(\frac{\Delta t}{\varepsilon^{2}}\right)\left\|\mathbf{U}^{1}\right\|_{3 h}^{2}+\Delta t \varepsilon^{2} \delta^{2} \eta^{2}\left|\mathbf{U}^{1}\right|_{1,3 h}^{2} \\
& -\frac{36 \Delta t^{2}}{3 \Delta x} \exp \left(\frac{2 \Delta t}{\varepsilon^{2}}\right)\left[\left(\left|\alpha_{1}\right|+\left|\alpha_{2}\right|\right)^{2}+\frac{4}{27 \Delta x^{3}}\left\|\mathbf{u}^{0}\right\|_{h}^{2}\right]\left\|\mathbf{u}^{0}\right\|_{h}^{2}\left|\mathbf{U}^{1}\right|_{1,3 h}^{2} \leq 0
\end{aligned}
$$

and using (4.22), we arrive at

$$
\left\|\mathbf{U}^{2}\right\|_{3 h}^{2}+\frac{\Delta t}{2} \varepsilon^{2} \delta^{2} \eta^{2}\left|\mathbf{U}^{1}\right|_{1,3 h}^{2} \leq \exp \left(\frac{\Delta t}{\varepsilon^{2}}\right)\left\|\mathbf{U}^{1}\right\|_{3 h}^{2} .
$$

We now assume that (4.30) holds true up to the order $q-1$, that is

$$
\left\|\mathbf{U}^{q}\right\|_{3 h}^{2}+\frac{\Delta t}{2} \varepsilon^{2} \delta^{2} \eta^{2}\left|\mathbf{U}^{q-1}\right|_{1,3 h}^{2} \leq \exp \left(\frac{\Delta t}{\varepsilon^{2}}\right)\left\|\mathbf{U}^{q-1}\right\|_{3 h}^{2} .
$$

and we observe that

$$
\left\|\mathbf{U}^{m+1}\right\|_{3 h}^{2} \leq \exp \left(\frac{\Delta t}{\varepsilon^{2}}\right)\left\|\mathbf{U}^{m}\right\|_{3 h}^{2}, \text { for } m=1, \ldots, q-1 .
$$

From (4.28) and (4.39) together with (4.22) we obtain the result. Thus using (4.3) and (4.17), we find

$$
\left\|\mathbf{u}^{m+1}\right\|_{h}^{2} \leq \exp \left(\frac{\Delta t}{\varepsilon^{2}}\right)\left\|\mathbf{u}^{m}\right\|_{h}^{2}, \text { for } m=0, \ldots, q .
$$

Assuming Eqs. (4.29) and (4.30) hold up to the order $n$, using the same approach as in the case $n=0$, we prove by induction on $r$ and $m$. Hence, (4.29) and (4.30) hold for any $n=s(q+1)$, where $s$ is a positive integer.

Therefore, the proof is complete.

Remark 4.4. By the subscript 3 h, we mean the discrete operators, discrete norms and discrete semi-norms are applied on the coarse mesh discretization.

Remark 4.5. To compare the stability regions of the multilevel method withe associated one level method, we use the step sizes $\frac{\Delta t}{p}$ and $\Delta x$ on the fine mesh and $\Delta t$ and $3 \Delta x$ on the coarser mesh for the one-level methods as discussed in this section. 
- When $p \leq 9$, the multilevel method has the same region of stability as the one-level method on the fine mesh but smaller region of stability from the one-level method considered on the coarse mesh.

- When $p \geq 81$, the multilevel method has the same region of stability as the one-level method on the coarse mesh but smaller region of stability from the one-level method considered on the fine mesh.

- When $9<p<81$, the multilevel method is less restrictive than the one-level method on the fine mesh and more restricted than the one-level method on the coarse mesh.

\section{Numerical results}

In this section, some numerical simulations of the $\mathrm{CCH}$ equation, (1.1), with specified initial condition and periodic boundary conditions at some values of $T$ are presented. All the results are computed in a matlab platform using Windows 8.1 Intel CORE i3, 6G RAM PC and the parameters are chosen as: $L=3, \varepsilon=0.3, p=5, \alpha_{1}=\alpha_{2}=\frac{1}{6}$ and $q=9$.

For the one-level finite volume method, we use the following temporal and spatial step sizes

- on the fine mesh:time step size $\Delta t / p$ and spatial step size $\Delta x$, i. e., $M=p M_{0}$ and $N=3 N_{0}$.

- on the coarse mesh: time step size $\Delta t$ and spatial step size $3 \Delta x$, i. e., $M=M_{0}$ and $N=N_{0}$.

For the implicit one-level method we use the following approximations:

$$
\tilde{\mathbf{u}}^{n}=\frac{1}{2}\left(\mathbf{u}^{n}+\mathbf{u}^{n-1}\right), \text { for } n=1,2, \ldots, M,
$$

and $\tilde{\mathbf{u}}^{0}=\mathbf{u}^{0}$.

In the case of multilevel methods, for $n=s(q+1)$, we consider the following approximations

$$
\begin{aligned}
\tilde{\mathbf{u}}^{s(q+1)+r / p} & =\frac{1}{2}\left(\mathbf{u}^{s(q+1)+r / p}+\mathbf{u}^{s(q+1)+(r-1) / p}\right), \text { for } r=1, \ldots, p-1, \\
\tilde{\mathbf{u}}^{s(q+1)} & =\mathbf{u}^{s(q+1)} \\
\tilde{\mathbf{U}}^{s(q+1)+m} & =\frac{1}{2}\left(\mathbf{U}^{s(q+1)+m}+\mathbf{U}^{s(q+1)+m-1}\right), \text { for } m=1, \ldots, q .
\end{aligned}
$$

\subsection{Example 1}

We use the initial condition

$$
u^{0}(x)=\sin \left(\frac{2 \pi x}{3}\right) .
$$

The numerical plots obtained from the four finite volume methods presented in sections 3 and 4 with this initial condition are shown in Figs 1 to 3. The CPU time for all the cases considered are shown by Tables 1-2. Figs 1 to 3 and Tables 1-2 show that the computing time and the results obtained using the multilevel method is intermediate between the results obtained using the one-level method on the fine mesh and the ones on the coarse mesh. For large domain, $L=30$, it is seen from Table $1 \mathrm{~b}$ that the computational time is greatly saved when using the implicit multilevel method as compared to the implicit one-level on the fine mesh. 


\begin{tabular}{|c|c|c|c|}
\hline Method & $\Delta x$ & $\Delta t$ & CPU time \\
\hline \multirow{3}{*}{ Fine } & 0.02 & 0.005 & 35.692 \\
\cline { 2 - 4 } & 0.1 & 0.005 & 1.212 \\
\cline { 2 - 4 } & 0.2 & 0.01 & 0.355 \\
\hline \multirow{3}{*}{ Coarse } & 0.02 & 0.005 & 1.971 \\
\cline { 2 - 4 } & 0.1 & 0.005 & 0.132 \\
\cline { 2 - 4 } & 0.2 & 0.01 & 0.089 \\
\hline \multirow{3}{*}{ Multilevel } & 0.02 & 0.005 & 19.221 \\
\cline { 2 - 4 } & 0.1 & 0.005 & 0.715 \\
\cline { 2 - 4 } & 0.2 & 0.01 & 0.243 \\
\hline
\end{tabular}

(a) $L=3$.

\begin{tabular}{|c|c|c|c|c|}
\hline Method & $\mathrm{T}$ & $\Delta x$ & $\Delta t$ & CPU time \\
\hline \multirow{3}{*}{ Fine } & 0.2 & 0.02 & 0.005 & out of memory (972.626) \\
\cline { 2 - 5 } & 1 & 0.1 & 0.005 & 34.484 \\
\cline { 2 - 5 } & 2 & 0.2 & 0.01 & 16.787 \\
\hline \multirow{3}{*}{ Coarse } & 0.2 & 0.02 & 0.005 & 11.556 \\
\cline { 2 - 5 } & 1 & 0.1 & 0.005 & 1.074 \\
\cline { 2 - 5 } & 2 & 0.2 & 0.01 & 1.355 \\
\hline \multirow{3}{*}{ Multilevel } & 0.2 & 0.02 & 0.005 & 233.720 \\
\cline { 2 - 5 } & 1 & 0.1 & 0.005 & 13.776 \\
\cline { 2 - 5 } & 2 & 0.2 & 0.01 & 6.506 \\
\hline
\end{tabular}

(b) $L=30$.

Table 1: CPU time for implicit methods at different values of $T$.

\begin{tabular}{|c|c|c|}
\hline $\mathrm{L}$ & Method & CPU time \\
\hline \multirow{3}{*}{3} & Fine & 0.158 \\
\cline { 2 - 3 } & Coarse & 0.049 \\
\cline { 2 - 3 } & Multilevel & 0.116 \\
\hline \multirow{3}{*}{30} & Fine & 1.180 \\
\cline { 2 - 3 } & Coarse & 0.124 \\
\cline { 2 - 3 } & Multilevel & 0.626 \\
\hline
\end{tabular}

Table 2: CPU time when $\Delta x=0.2$ and $\Delta t=0.005$ for the explicit methods at $T=2$.

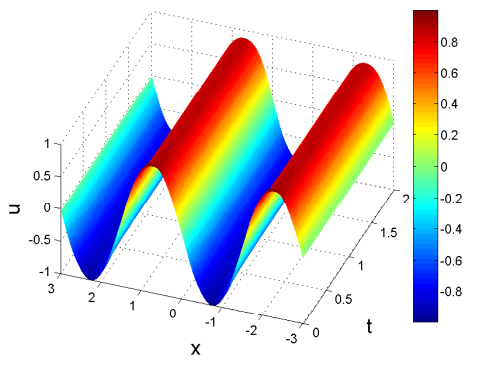

(a) One-level method on the fine mesh

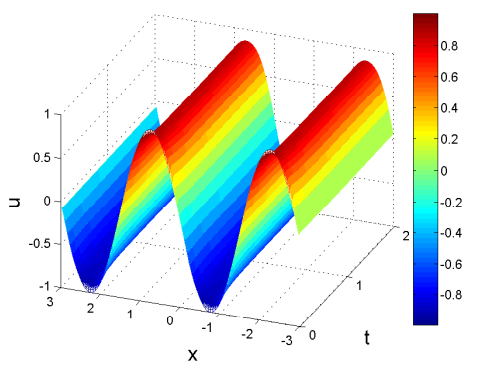

(c) One-level method on the coarse mesh

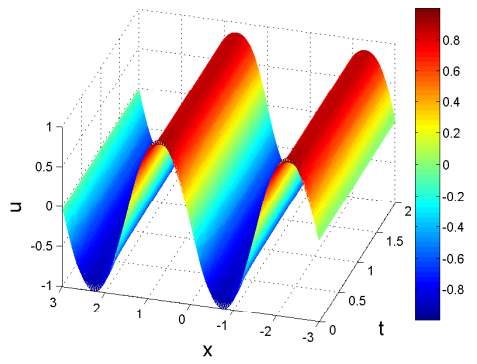

(b) Multilevel Method

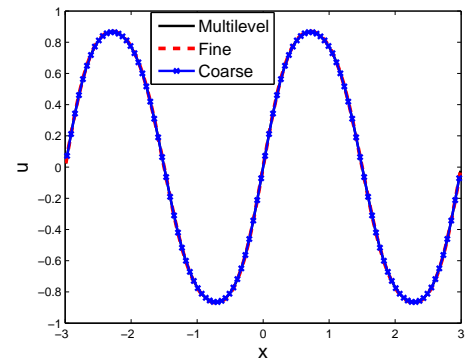

(d) $u$ versus $x$ at $T=2$

Figure 1: Comparison of the implicit methods when $\Delta x=0.02$ and $\Delta t=0.005$ for example 1 at $T=2$ 


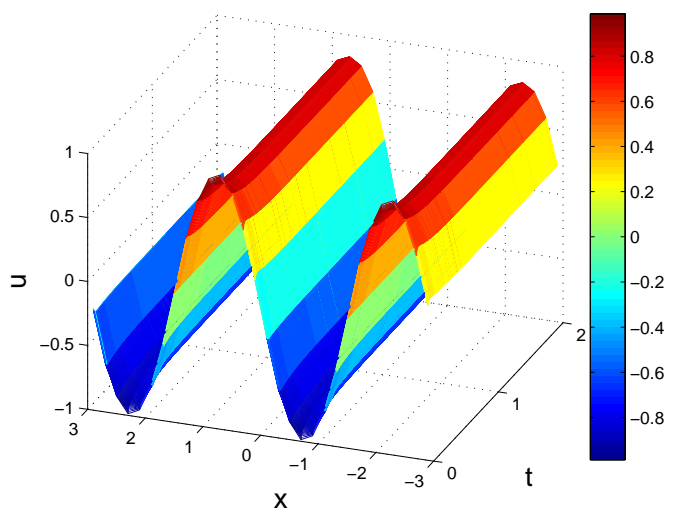

(a) One-level method on the fine mesh

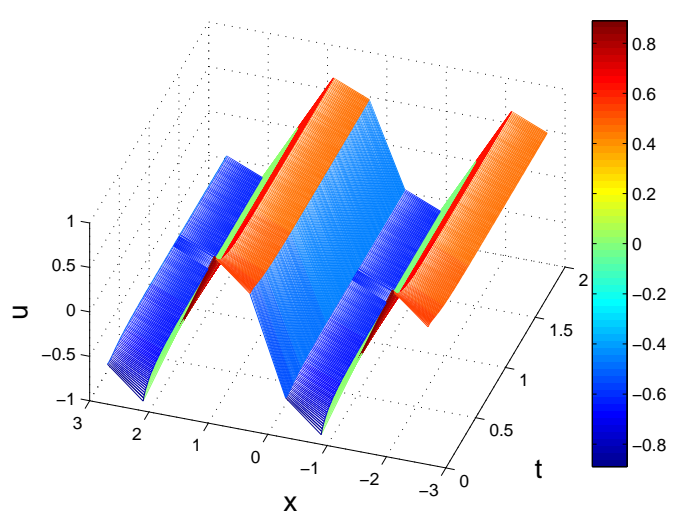

(c) One-level method on the coarse mesh

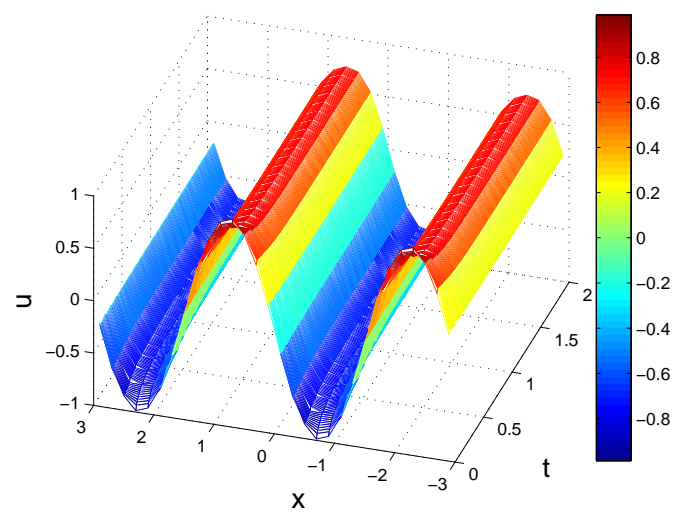

(b) Multilevel Method

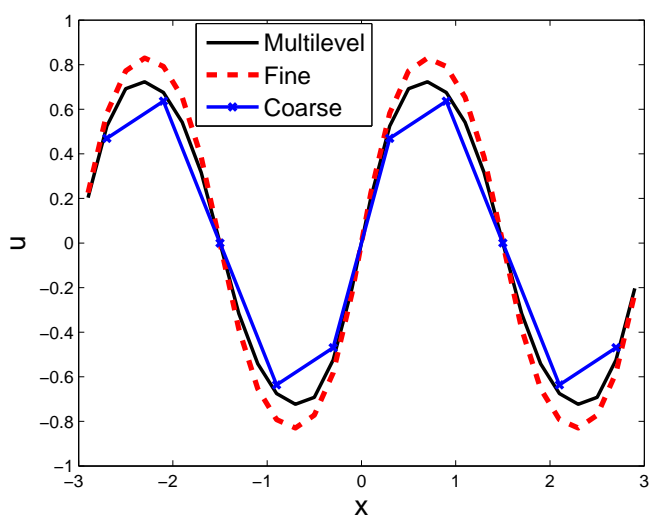

(d) $u$ versus $x$ at $T=2$

Figure 2: Comparison of the implicit methods when $\Delta x=0.2$ and $\Delta t=0.01$ for example 1 at $T=2$ 


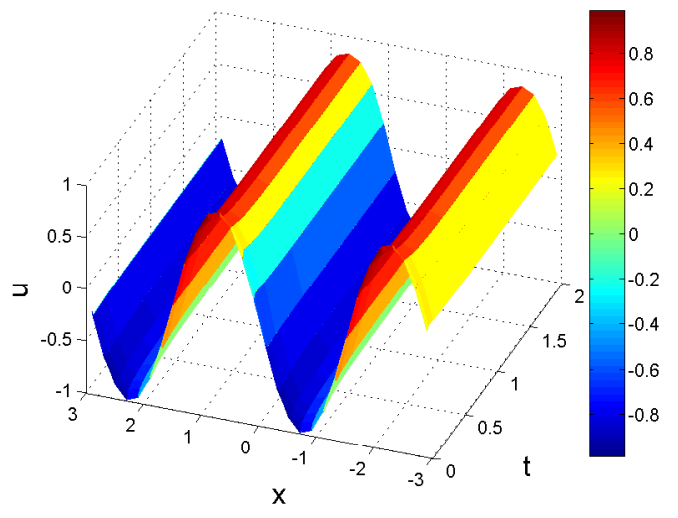

(a) One-level method on the fine mesh

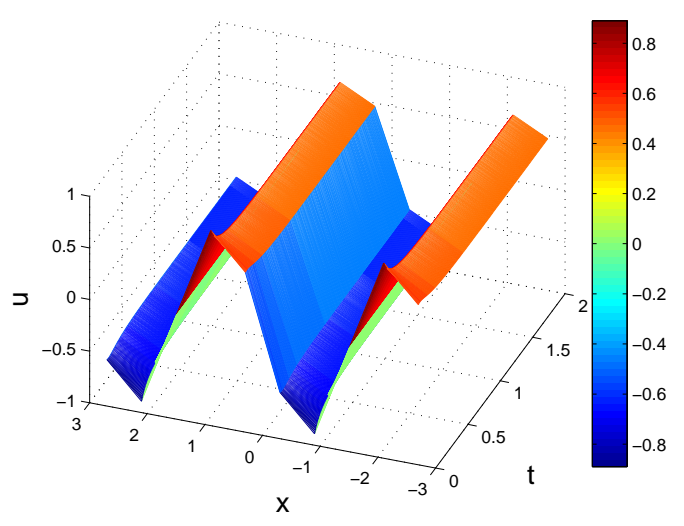

(c) One-level method on the coarse mesh

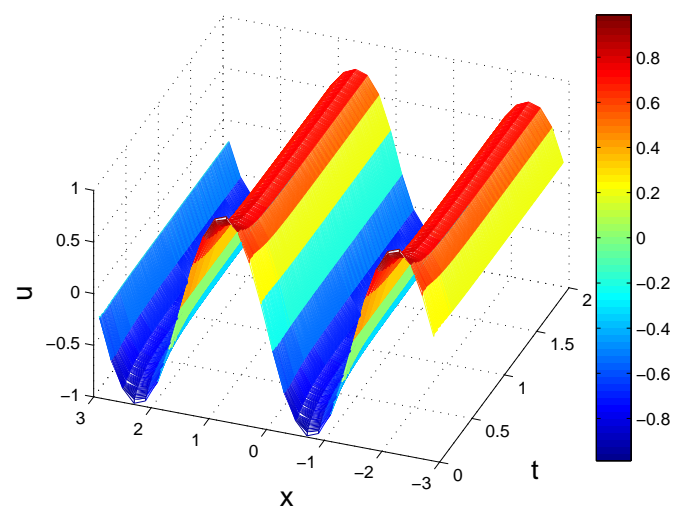

(b) Multilevel Method

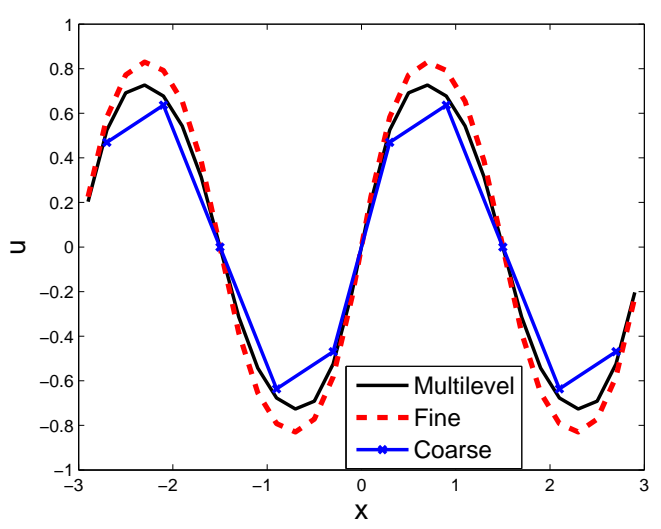

(d) $u$ versus $x$ at $T=2$

Figure 3: Comparison of the explicit methods when $\Delta x=0.2$ and $\Delta t=0.005$ for example 1 at $T=2$ 


\subsection{Example 2}

We consider the initial condition

$$
u^{0}(x)=\cos (\pi x)
$$

with periodic boundary conditions.

The numerical simulations obtained from the finite volume methods are shown by Figs 4 to 6 . As in example 1, the results obtained from the multilevel method are intermediate between the results from the one-level on the fine mesh and that of the ones on the coarse mesh.

\subsection{Convergence rate}

Here, we consider the exact solution

$$
u(x, t)=\sin \left(\frac{2 \pi x}{L}\right) \cos (2 \pi t),
$$

for which the source term is obtained on substitution of $u$ into Eq. (1.1).

The convergence rate is calculated based on the relation:

$$
\text { Rate }=\log \left(e_{1} / e_{2}\right) / \log (2),
$$

where $e_{1}$ and $e_{2}$ are $L_{2}$-errors when the spatial step sizes are $\Delta x$ and $\Delta x / 2$, respectively. Tables 3 and 4 show the $L_{2}$-errors and the corresponding convergence rates due to each of the finite volume methods for some temporal and spatial step sizes at $T=0.1$. It is shown that all the methods are second order accurate in space. The numerical simulations of the exact solution and the corresponding numerical solutions are also shown by Figs 8 and 9 .

The $L_{2}$-error for the multilevel methods is calculated by the formula:

$$
L_{2} \text {-error }=\sqrt{\Delta x \sum_{i=1}^{3 N_{0}}\left(u_{i}^{M_{0}}-v_{i}^{M_{0}}\right)^{2}},
$$

where $u_{i}^{M_{0}}$ and $v_{i}^{M_{0}}$ are numerical and exact solutions, respectively. Fig. 7 shows the 2D plot of $q$ versus $L_{2}$-error obtained using the multilevel method for some values of $\Delta x$ and $\Delta t$. Let $m$ be an integer and $0 \leq s<1$ such that

$$
M_{0}-1=m(q+1)+s(q+1) .
$$

In Fig 7, the points, $\left(q, L_{2}\right.$-error $(q)$ ), in which $s=0$ (as in Fig 7b) or $s$ is small as compared to the values of $s$ for some neighbouring $q$ 's (as in Fig 7a), are connected by a curve. It is shown from these figures that the curve is increasing and all the $L_{2}$-errors obtained using the multilevel methods lie above these curves. Thus we note that the accuracy of the multilevel method relies on suitable choice of $q$. 


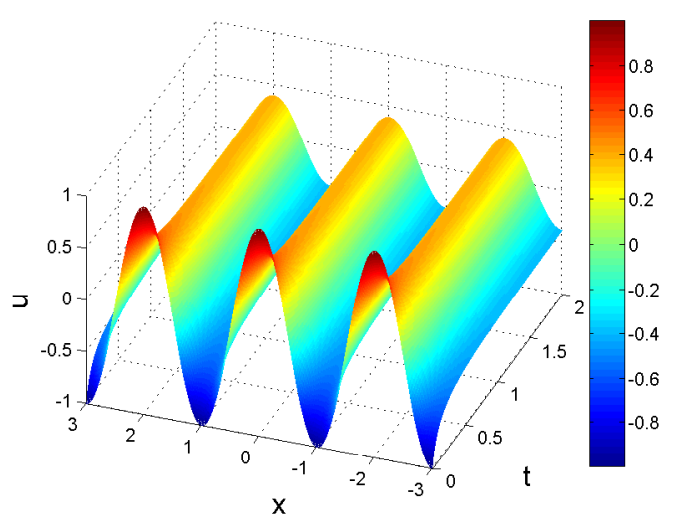

(a) One-level method on the fine mesh

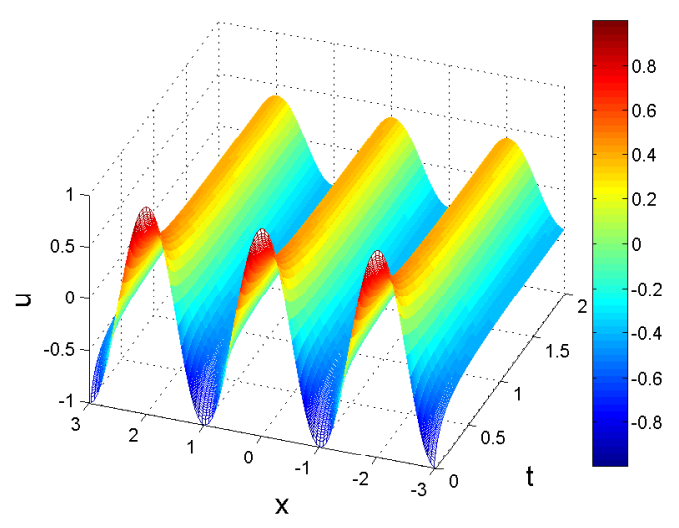

(c) One-level method on the coarse mesh

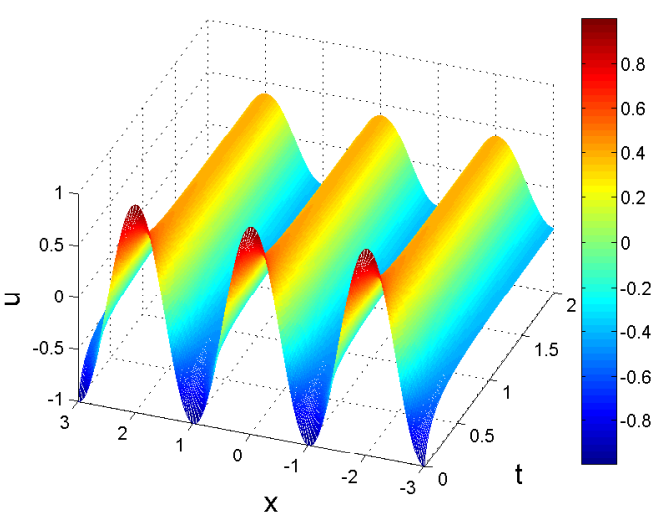

(b) Multilevel Method

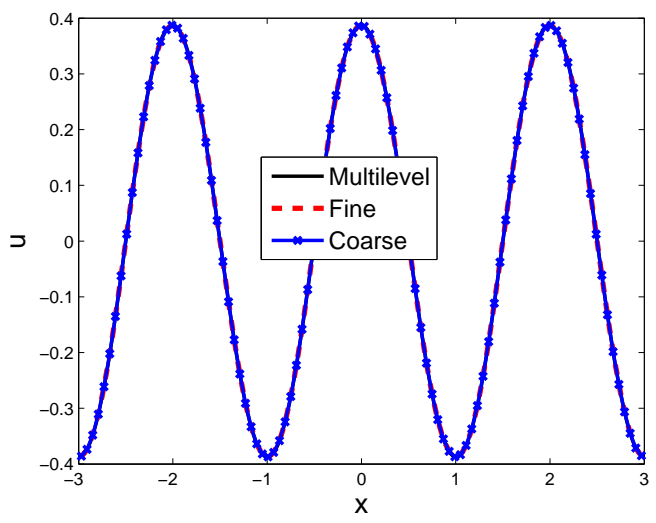

(d) $u$ versus $x$ at $T=2$

Figure 4: Comparison of implicit methods when $\Delta x=0.02$ and $\Delta t=0.005$ for example 2 at $T=2$

\begin{tabular}{|c|c|c|c|c|c|}
\hline Method & $\Delta x$ & $\Delta t$ & $L_{2}$ error & Rate & CPU time \\
\hline \multirow{4}{*}{ Fine } & 0.2 & 0.01 & 0.0432 & & \\
\cline { 2 - 6 } & 0.1 & 0.0025 & 0.0111 & 1.9606 & 0.406 \\
\cline { 2 - 6 } & 0.05 & 0.000625 & 0.0028 & 1.9889 & 3.115 \\
\cline { 2 - 6 } Coarse & 0.025 & 0.00015625 & $6.9982 \times 10^{-4}$ & 1.9971 & 24.898 \\
\hline \multirow{4}{*}{ Multilevel } & 0.2 & 0.01 & 0.4077 & & \\
\cline { 2 - 6 } & 0.1 & 0.0025 & 0.0872 & 2.2250 & 0.117 \\
\cline { 2 - 6 } & 0.05 & 0.000625 & 0.0228 & 1.9370 & 0.282 \\
\cline { 2 - 6 } & 0.025 & 0.00015625 & 0.0058 & 1.9743 & 1.631 \\
\cline { 2 - 6 } & 0.2 & 0.01 & 0.3521 & & \\
\cline { 2 - 6 } & 0.05 & 0.0025 & 0.0623 & 2.4984 & 0.261 \\
\hline & 0.025 & 0.00015625 & 0.0151 & 2.0467 & 0.978 \\
\hline
\end{tabular}

Table 3: $L_{2}$-error and convergence rate of implicit methods for some values of $\Delta t$ and $\Delta x$ at $T=0.1$ 


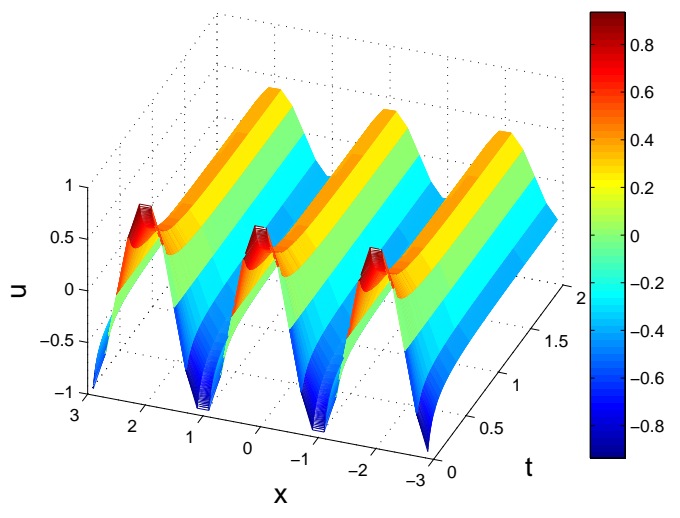

(a) One-level method on the fine mesh

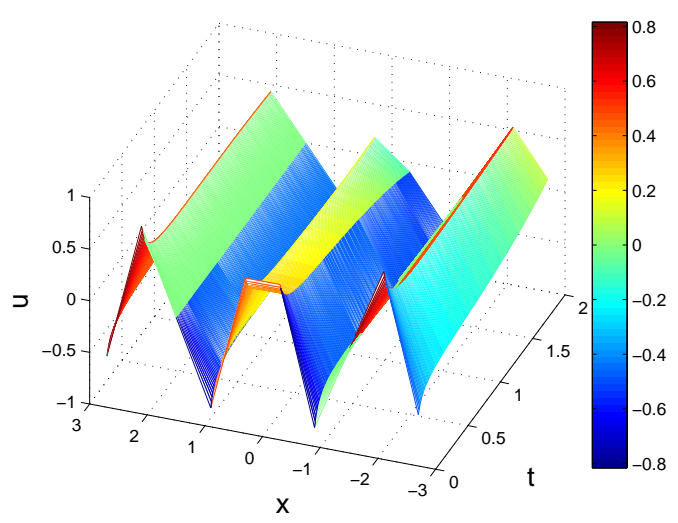

(c) One-level method on the coarse mesh

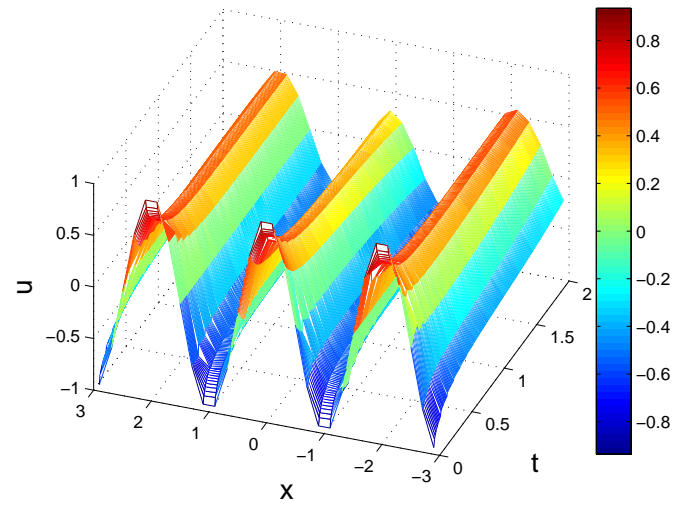

(b) Multilevel Method

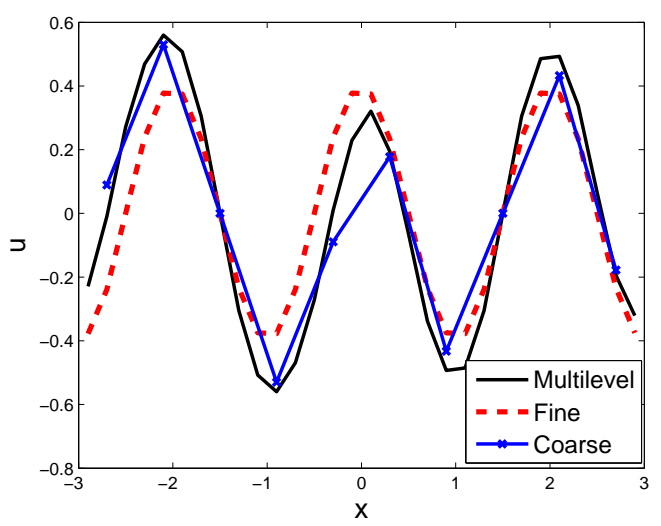

(d) $u$ versus $x$ at $T=2$

Figure 5: Comparison of implicit methods when $\Delta x=0.2$ and $\Delta t=0.01$ for example 2 at $T=2$

\begin{tabular}{|c|c|c|c|c|c|}
\hline Method & $\Delta x$ & $\Delta t$ & $L_{2}$ error & Rate & CPU time \\
\hline \multirow{3}{*}{ Fine } & 0.2 & 0.002 & 0.0348 & & \\
\cline { 2 - 6 } & 0.1 & 0.00025 & 0.0091 & 1.9364 & 2.871 \\
\cline { 2 - 6 } & 0.05 & 0.00003125 & 0.0023 & 1.9774 & 45.084 \\
\hline \multirow{3}{*}{ Coarse } & 0.2 & 0.002 & 0.3946 & & \\
\cline { 2 - 6 } & 0.1 & 0.00025 & 0.0778 & 2.3418 & 0.331 \\
\cline { 2 - 6 } Multilevel & 0.05 & 0.00003125 & 0.0204 & 1.9327 & 3.171 \\
\cline { 2 - 6 } & 0.2 & 0.002 & 0.1485 & & \\
\cline { 2 - 6 } & 0.05 & 0.00025 & 0.0287 & 2.3730 & 0.794 \\
\hline
\end{tabular}

Table 4: $L_{2}$-error and convergence rate of explicit methods for some values of $\Delta t$ and $\Delta x$ at $T=0.1$ 


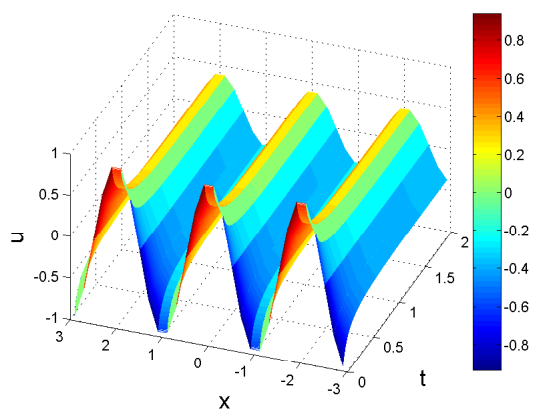

(a) One-level method on the fine mesh

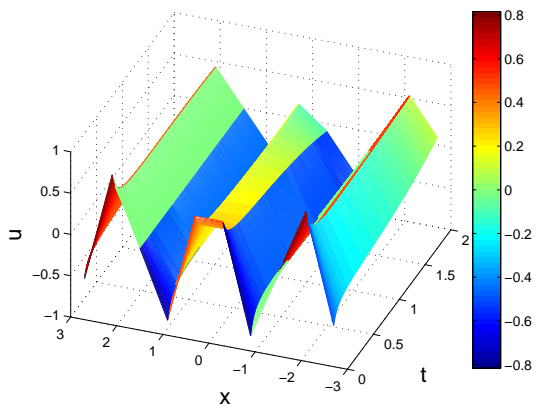

(c) One-level method on the coarse mesh

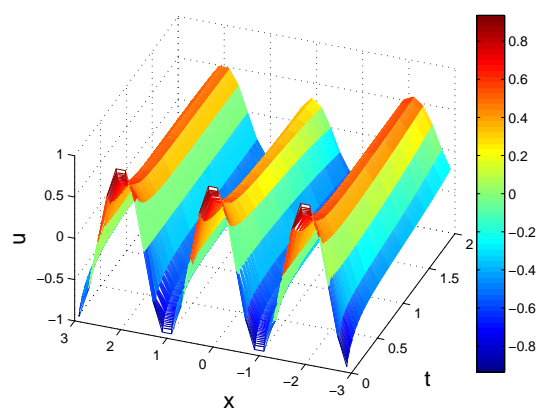

(b) Multilevel Method

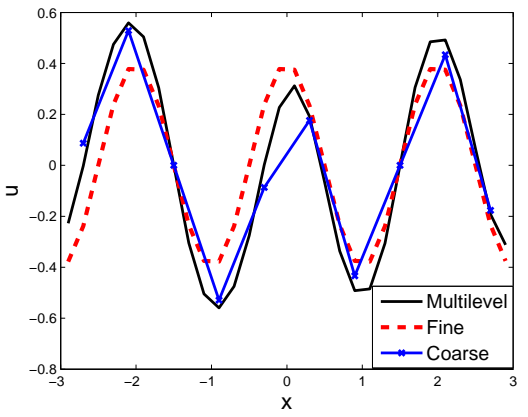

(d) $u$ versus $x$ at $T=2$

Figure 6: Comparison of explicit methods when $\Delta x=0.2$ and $\Delta t=0.005$ for example 2 at $T=2$

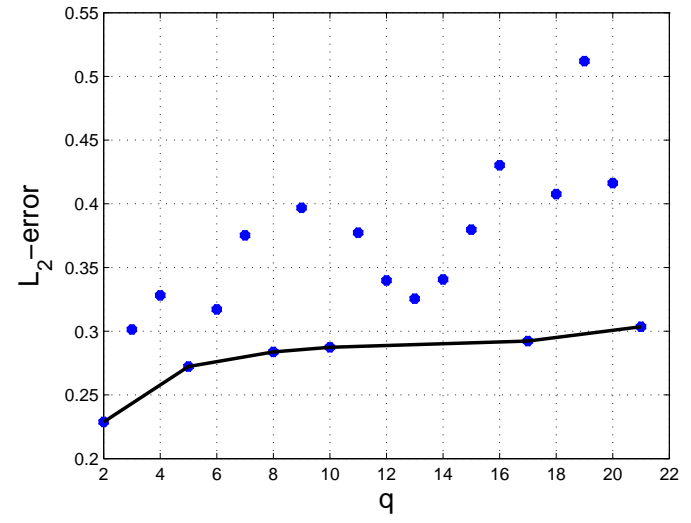

(a) Implicit multilevel when $\Delta x=0.2, \Delta t=0.01$ at $T=2$.

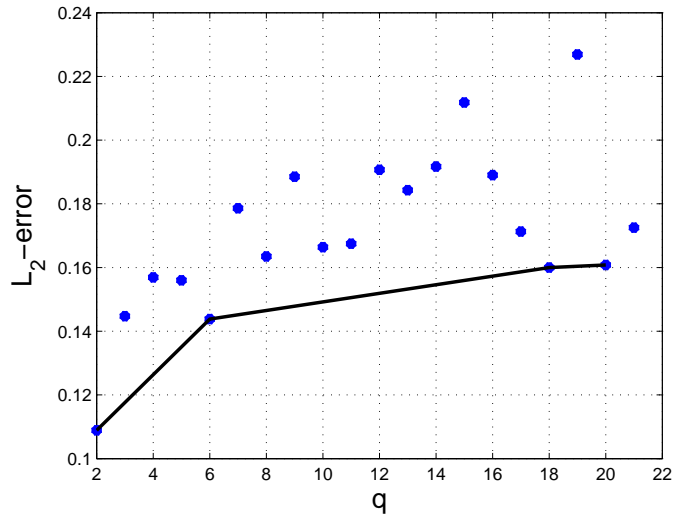

(b) Explicit multilevel when $\Delta x=0.2, \Delta t=0.005$ at $T=2$.

Figure 7: $q$ versus $L_{2}$-error for multilevel methods 


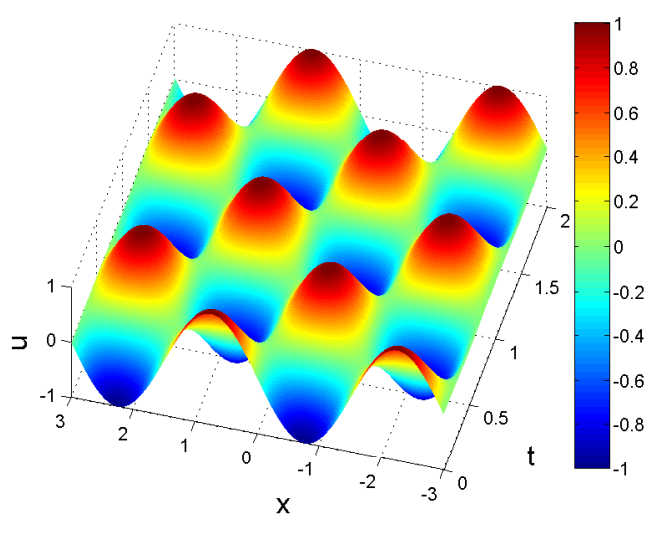

(a) Exact

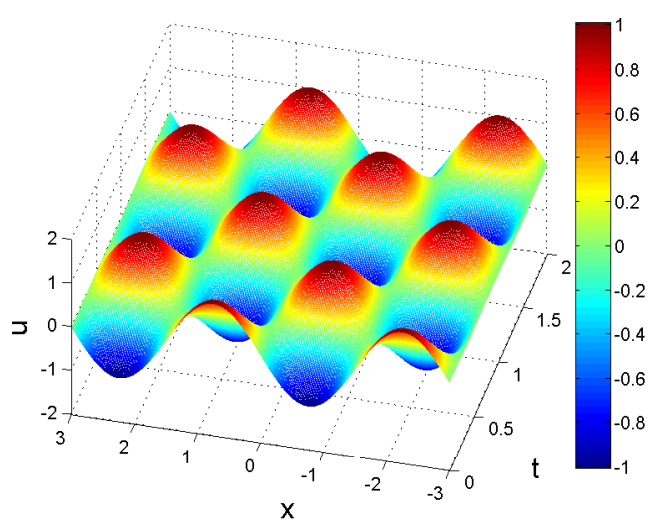

(c) Multilevel method

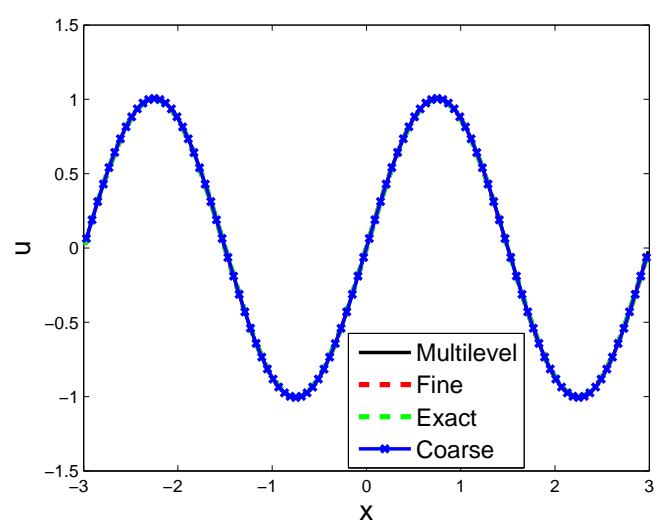

(e) Implicit methods at $T=2$

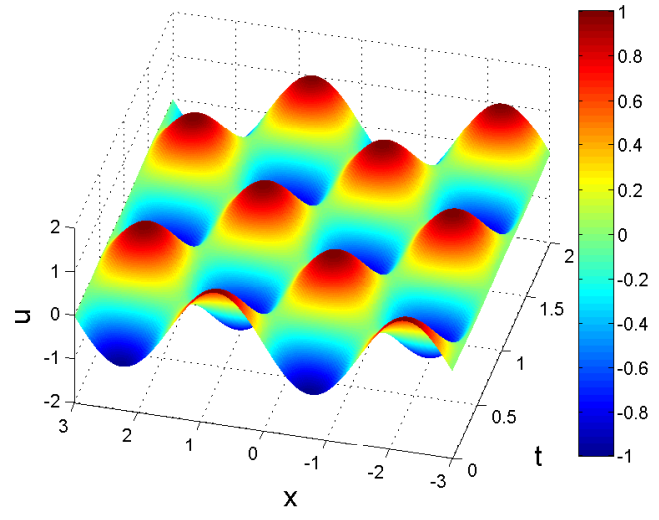

(b) One-level on the fine mesh

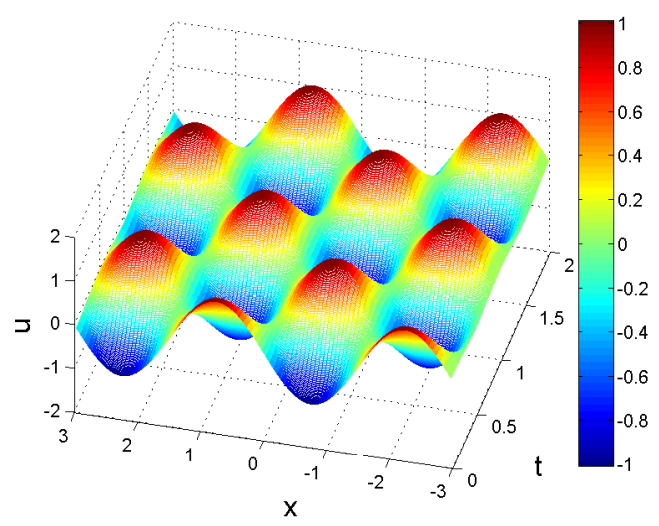

(d) One-level on the coarse mesh

Figure 8: Numerical results from implicit one level and multilevel methods when $\Delta t=0.005$ and $\Delta x=0.02$ 


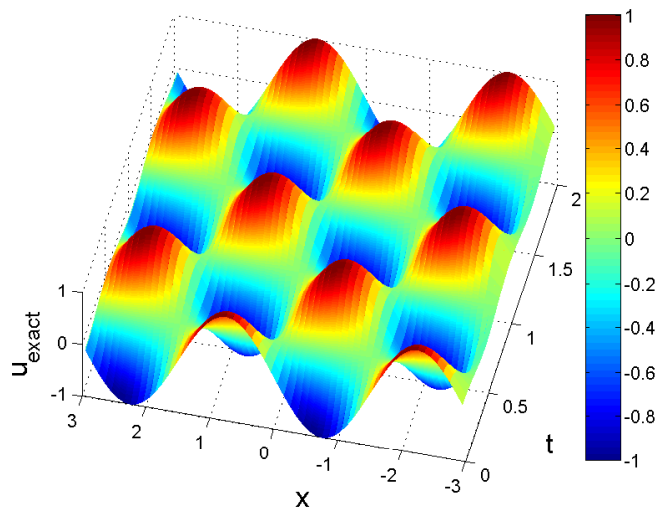

(a) Exact

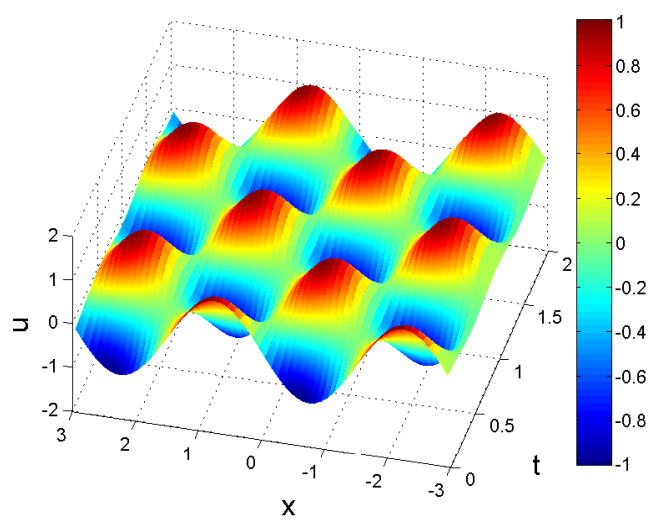

(c) Multilevel method

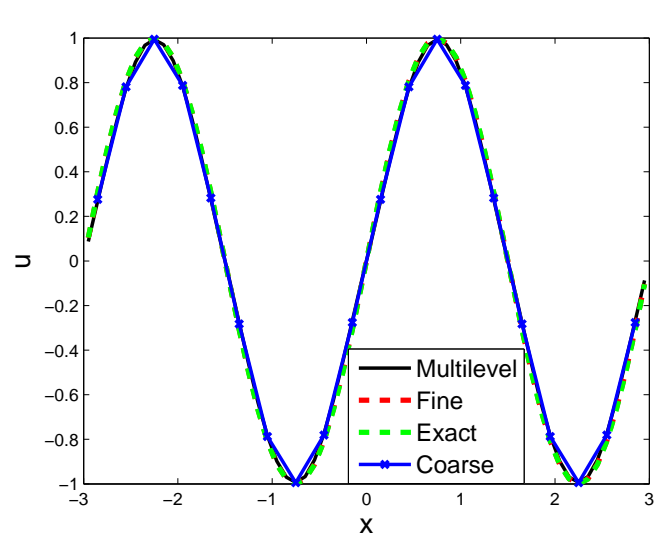

(e) Explicit methods at $T=2$

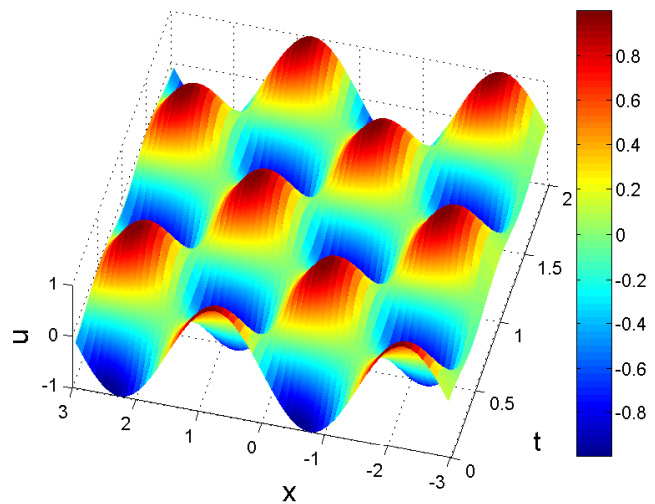

(b) One-level on the fine mesh

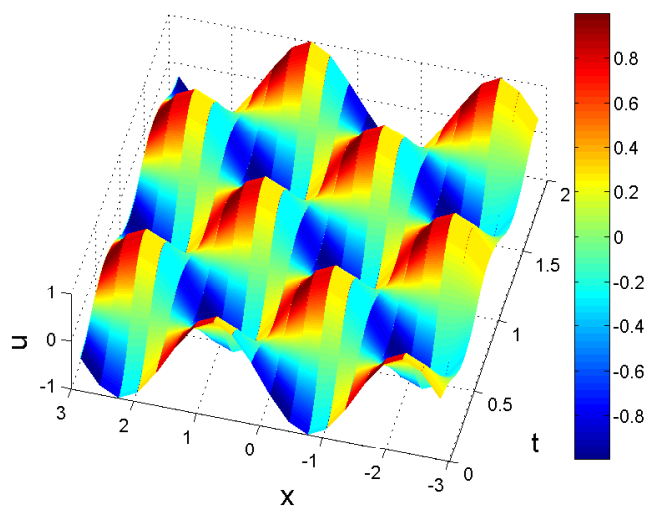

(d) One-level on the coarse mesh

Figure 9: Numerical results from explicit one level and multilevel methods when $\Delta t=0.0005$ and $\Delta x=0.1$ 


\section{Conclusion}

In this work, we presented one-level and multilevel finite volume approximations for the solution of the convective Cahn-Hilliard equation with given initial condition and periodic boundary conditions. We study the existence/uniqueness of solutions, stability and convergence analysis of the finite volume methods. We computed the computational time for some chosen temporal and spatial step sizes at some values of $T$. It is observed that the multilevel method is faster than the one-level. When the domain is large, the multilevel methods saves more time as compared to the onelevel method. From the convergence analysis, we prove that all methods are second order accurate in space and it is validated numerically. Our next task is to extend this analysis to multi-dimensional problems.

\section{Acknowledgments}

A.R. Appadu is grateful to the DST/NRF SARChI Chair in Mathematical Models and Methods in Bioengineering and Biosciences and the National Research Foundation of South Africa Grant N0. 95864 for funding. J.K. Djoko is funded through the incentive fund N00 401 Project 85796. H. H. Gidey is grateful to the University of Pretoria, African Institute for Mathematical Sciences (AIMS)-South Africa and Aksum University (Ethiopia) for their financial support for his $\mathrm{PhD}$ studies.

\section{References}

[1] A. A. Aderogba, M. Chapwanya and J. K. Djoko, On fractional step-splitting scheme for the Cahn-Hilliard equation, Engineering Computations, 31, Iss 7, 1151-1168, 2014.

[2] A. Eden and V. K. Kalantarov, The convective Cahn-Hilliard equation, Applied Mathematics Letters, 20, 455$461,2007$.

[3] J. K. Djoko, On the long-time stability of a backward euler scheme for Burgers' Equation with Polynomial Force, Numerical Methods for Partial Differential Equations, 24, 1371-1387, 2008.

[4] A. Bousquet, M. Marion and R. Temam, Finite volume multilevel approximation of the shallow water equations with a time explicit scheme, International Journal of Numerical Analysis and Modeling, 11, no 4, 762-786, 2014.

[5] S. J. Watson, F. Otto, B. Y. Rubinstein and S. H. Davis, Coarsening dynamics of the convective Cahn-Hilliard equation, Physica D, 178, 127-148, 2003.

[6] M. A. Zaks , A. Podolny, A. A. Nepomnyashchy and A. A. Golovin, Periodic stationary patterns governed by a convective Cahn-Hilliard equation, SIAM Journal of Applied Mathematics, 66 No 2, 700-720, 2006.

[7] A. A. Golovin, A. A. Nepomnyashchy, S. H. Davis and M. A. Zaks, Convective Chan-Hilliard models: From Coarsening to Roughening, Physical Review Letters, 86 No 8, 1550-1553, 2001.

[8] A. Podolny, M. A. Zaks, B. Y. Rubinstein, A. A. Golovin and A. A. Nepomnyashchy, Dynamics of domin walls governed by the convective Cahn-Hilliard equation, Physica D, 201, 291-305, 2005.

[9] X. Zhao and C. Liu, Optimal control of the Convective Cahn-Hilliard Equation, Applicable Analysis, 92, No. 5, 1028-1045, 2013.

[10] X. Zhao and C. Liu, Optimal control of the convective Cahn-Hilliard equation in 2D case, Applied Mathematics and Optimization, 70, Issue 1, 61-82, 2014.

[11] S.J. Watson, Crystal Growth, Coarsening and the convective Cahn-Hilliard equation, International Series of Numerical Mathematics, 147, 329-341, 2003.

[12] N. Khiari, T. Achouri, M. L. Ben Mohamed and K. Omrani, Finite Difference Approximations for the CahnHilliard Equations, Numerical methods for partial differential equations, 23,437-455, 2007. 
[13] A. Bousquet and R. Temam, A finite volume multilevel approximation of the Shallow-Water equations, European Conference on Computational Fluid Dynamics ECCOMAS CFD 2010, V, Lisbon, Portugal, 14-17 June 2010.

[14] K. Adamy, A. Bousquet, S. Faure, J. Lamine and R. Temam, A multilevel method for finite volume discretization of the two-dimensional nonlinear shallow-water equations, Ocean Modeling, 33, 235-256, 2010.

[15] R. Temam, Navier-Stokes Equations: Theory and Numerical Analysis, Vol. 2, North-Holland, 1979.

[16] C. Folas, O. Manley and R. Temam, On the interaction of small and large eddles in two-dimensional turbulant flows, Mathematical modelinc and numerical Analysis, 22, 99-114, 1988.

[17] M. Marion and R. Temam, Nonlinear Galerkin methods, SIAM Journal of Numerical Analysis, 26(5), 1139$1157,1989$.

[18] R. Temam, Inertial manifolds and multigrid methods, SIAM Journal of Mathematical Analysis, 21(1), 154-178, 1990.

[19] A. Bousquet, M. Marion and R. Temam, Finite volume multilevel approximation of the shallow water equations, Chinese Annals of Mathematics, Series B, 34, Issue 1, 1-28, 2013,

[20] M. Chen and R. Temam, Incremental unknowns in finite differences: condition number of the matrix, SIAM journal of Matrix analysis application, 14(2), 432-455, 1993.

[21] K. Leung, Theory of morphological instability in driven systems, Statistical Physics 61 (1/2), 345-364, 1990.

[22] C. L. Emmott, A. J. Bray, Coarsening dynamics of a one-dimensional driven Cahn-Hilliard system, Physical Review E, 54 (5), 4568-4575, 1996.

[23] R. E. Mickens, Nonstandard Finite Difference Models of Differential Equations, World Scientific, Singapore, 1994.

[24] R. Anguelov, J. K. Djoko, and J. M-S. Lubuma, Energy properties preserving finite difference schemes for Burgers equation, Numer Methods Partial Differential Equations (2008), 24, 41-59.

[25] V. Girault and P. A. Raviart, Finite element methods for Navier-Stokes equations: theory and algorithms. Vol. 5. Springer Science \& Business Media, 2012.

[26] R. J. LeVeque, Finite volume methods for hyperbolic problems. Vol. 31. Cambridge university press, 2002. 